\title{
Butterfly Effects Arising from Starting Materials in Fused-Ring Electron Acceptors
}

Tengfei Li,${ }^{\dagger}$ Yao Wu, ${ }^{\ddagger}$ Jiadong Zhou, ${ }^{\S}$ Mengyang Li, ${ }^{\|}$Jingnan $\mathrm{Wu},{ }^{\perp}$ Qin $\mathrm{Hu},{ }^{\ddagger}, \#$ Boyu Jia, ${ }^{\dagger}$ Xiran Pan,${ }^{\dagger}$ Maojie Zhang, ${ }^{\perp}$ Zheng Tang, ${ }^{\|}$Zengqi Xie, ${ }^{\S}$ Thomas P. Russell, ${ }^{\ddagger, \#}$ and Xiaowei Zhan*,†

${ }^{\dagger}$ Department of Materials Science and Engineering, College of Engineering, Key Laboratory of Polymer Chemistry and Physics of Ministry of Education, Peking University, Beijing 100871, China

${ }^{\star}$ Department of Polymer Science and Engineering, University of Massachusetts Amherst, 120 Governors Drive, Amherst, Massachusetts 01003, United States

${ }^{\S}$ Institute of Polymer Optoelectronic Materials and Devices, State Key Laboratory of Luminescent Materials and Devices, South China University of Technology, Guangzhou 510640, China

"Center for Advanced Low-Dimension Materials, State Key Laboratory for Modification of Chemical Fibers and Polymer Materials, College of Materials Science and Engineering, Donghua University, Shanghai 201620, China

${ }^{\perp}$ Laboratory of Advanced Optoelectronic Materials, College of Chemistry, Chemical Engineering and Materials Science, Soochow University, Suzhou 215123, China

${ }^{\#}$ Materials Sciences Division, Lawrence Berkeley National Laboratory, 1 Cyclotron Road, Berkeley, California 94720, United States 


\section{Materials}

Unless stated otherwise, all the solvents and chemical reagents used were obtained commercially and used without further purification. Toluene and THF were distilled from sodium benzophenone under nitrogen before use. Compound 1 was purchased from Accela ChemBio Co., Ltd. Compound 3 was purchased from Aladdin Inc. TT-AB, SM2 and 2FIC were purchased from Suna Tech Inc. SM1 and PM6 were synthesized according to the literature procedures. ${ }^{\mathrm{S} 1, \mathrm{~S} 2}$

\section{Synthesis}
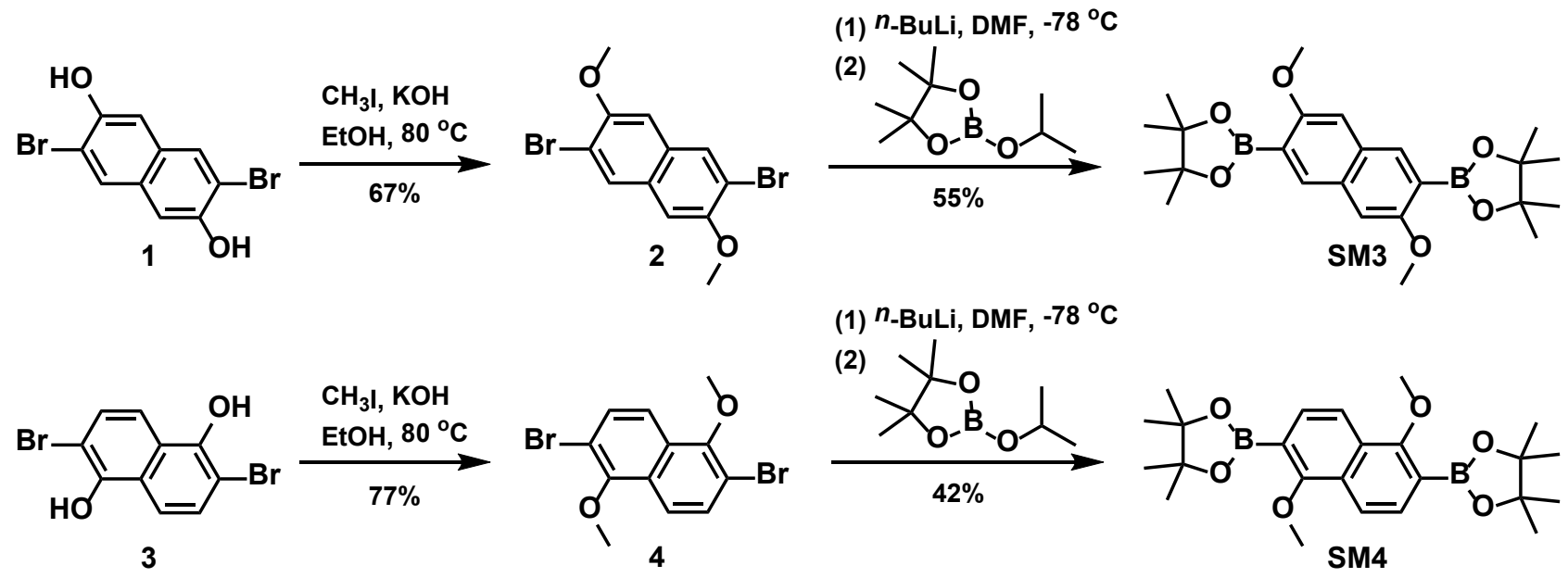

Scheme S1. Synthetic routes for SM3 and SM4

Compound 2. A solution of compound 1 ( $1 \mathrm{~g}, 3.15 \mathrm{mmol})$ and $\mathrm{KOH}(530 \mathrm{mg}, 9.45 \mathrm{mmol})$ in ethanol $(20 \mathrm{~mL})$ was deoxygenated with nitrogen for $15 \mathrm{~min} . \mathrm{CH}_{3} \mathrm{I}(0.6 \mathrm{~mL}, 9.52 \mathrm{mmol})$ was added dropwise, and the solution was refluxed for $12 \mathrm{~h}$ and then cooled down to room temperature. The mixture was filtered and the residue was washed with water $(2 \times 20 \mathrm{~mL})$ and purified by column chromatography on silica gel using petroleum ether/dichloromethane (4:1) as eluent yielding a white solid (736 mg, 67\%). ${ }^{1} \mathrm{H}$ NMR (400 MHz, $\left.\mathrm{CDCl}_{3}\right): \delta 7.90$ (s, 2H), 6.99 (s, 2H), 3.92 (s, 6H). ${ }^{13} \mathrm{C}$ NMR (100 MHz, $\left.\mathrm{CDCl}_{3}\right): \delta 152.67,130.85,129.30,114.19,105.78,56.22$. MS (MALDI-TOF): $\mathrm{m} / z$ 346.0 $\left(\mathrm{M}^{+}\right)$. Anal. calc. for $\mathrm{C}_{12} \mathrm{H}_{10} \mathrm{Br}_{2} \mathrm{O}_{2}$ : C, 41.65; H, 2.91. Found: $\mathrm{C}, 41.58 ; \mathrm{H}, 2.99$. 
SM3. To a solution of compound $2(692 \mathrm{mg}, 2 \mathrm{mmol})$ in THF $(30 \mathrm{~mL})$ at $-78{ }^{\circ} \mathrm{C}$ was added 2.4 $\mathrm{M} n$-butyllithiumin in hexane $(4.2 \mathrm{~mL}, 10 \mathrm{mmol})$ dropwise under nitrogen. The mixture was stirred at $-78{ }^{\circ} \mathrm{C}$ for $2 \mathrm{~h}$, and then 2-isopropoxy-4,4,5,5-tetramethyl-1,3,2-dioxaborolane (1.9 $\left.\mathrm{g}, 10 \mathrm{mmol}\right)$ was added. The mixture was stirred overnight at room temperature. Brine $(30 \mathrm{~mL})$ was added and the mixture was extracted with dichloromethane $(2 \times 30 \mathrm{~mL})$. The organic phase was dried over anhydrous $\mathrm{MgSO}_{4}$ and filtered. After removing the solvent from the filtrate, the residue was purified by column chromatography on silica gel using petroleum ether/dichloromethane $(1: 2)$ as the eluent yielding a gray solid (485 mg, 55\%). ${ }^{1} \mathrm{H}$ NMR (400 MHz, $\left.\mathrm{CD}_{2} \mathrm{Cl}_{2}\right): \delta 8.08(\mathrm{~s}, 2 \mathrm{H}), 7.07(\mathrm{~s}, 2 \mathrm{H}), 3.88$ (s, 6H). 1.39 (s, 24H). ${ }^{13} \mathrm{C}$ NMR (100 MHz, $\left.\mathrm{CDCl}_{3}\right): \delta 159.49,130.92,128.18,118.94,105.41,83.73$, 55.73, 24.89. MS (MALDI-TOF): $m / z 440.3\left(\mathrm{M}^{+}\right)$.

Compound 4. This compound was prepared following the same procedure as for compound 2 . The product was afforded as a white solid (846 mg, 77\%). ${ }^{1} \mathrm{H}$ NMR (400 $\left.\mathrm{MHz}, \mathrm{CDCl}_{3}\right): \delta 7.78(\mathrm{~d}, J$ $=8.8 \mathrm{~Hz}, 2 \mathrm{H}), 7.62(\mathrm{~d}, J=8.8 \mathrm{~Hz}, 2 \mathrm{H}), 4.40(\mathrm{~s}, 6 \mathrm{H}) .{ }^{13} \mathrm{C} \mathrm{NMR}\left(100 \mathrm{MHz}, \mathrm{CDCl}_{3}\right): \delta 153.70,131.29$, 129.87, 119.61, 113.75, 61.68. MS (MALDI-TOF): $m / z$ 346.0 $\left(\mathrm{M}^{+}\right)$. Anal. calc. for $\mathrm{C}_{12} \mathrm{H}_{10} \mathrm{Br}_{2} \mathrm{O}_{2}$ : C, 41.65; H, 2.91. Found: C, 41.61; H, 2.92.

SM4. This compound was prepared following the same procedure as for SM3. The product was afforded as a gray solid (370 mg, 42\%). ${ }^{1} \mathrm{H}$ NMR (400 MHz, $\left.\mathrm{CD}_{2} \mathrm{Cl}_{2}\right): \delta 7.89(\mathrm{~d}, J=8.4 \mathrm{~Hz}, 2 \mathrm{H})$, $7.69(\mathrm{~d}, J=8.4 \mathrm{~Hz}, 2 \mathrm{H}), 3.96(\mathrm{~s}, 6 \mathrm{H}) .1 .37(\mathrm{~s}, 24 \mathrm{H}) .{ }^{13} \mathrm{C}$ NMR $\left(100 \mathrm{MHz}, \mathrm{CDCl}_{3}\right): \delta 163.46,131.36$, 131.23, 117.64, 83.77, 63.40, 24.68. MS (MALDI-TOF): $m / z 440.3\left(\mathrm{M}^{+}\right)$. Anal. calc. for $\mathrm{C}_{24} \mathrm{H}_{34} \mathrm{~B}_{2} \mathrm{O}_{6}$ : C, 65.49; H, 7.79. Found: C, 65.54; H, 7.66. 


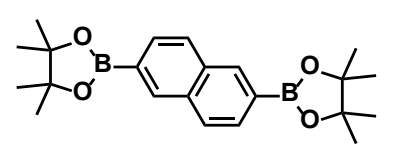

SM1

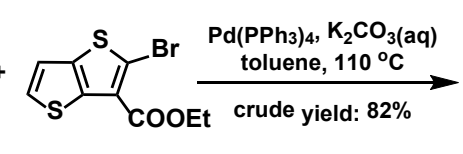

TT-AB

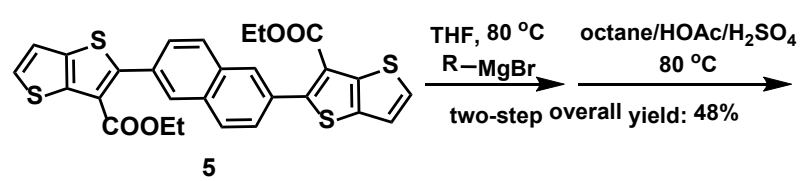

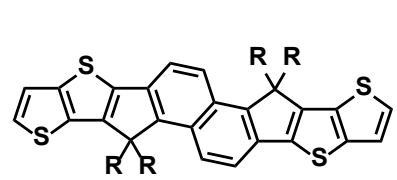

NO

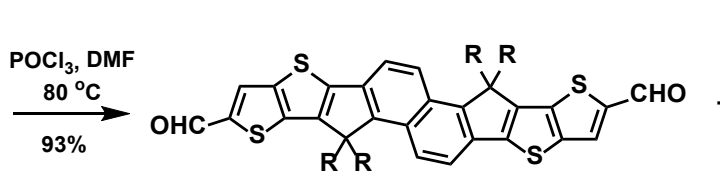

NO-CHO

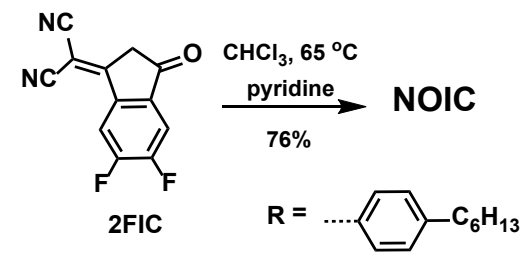

Scheme S2. Synthetic route for NOIC

Compound 5. To a three-necked round bottom flask were added SM1 (570 mg, $1.5 \mathrm{mmol}$ ), TT-AB (873 mg, $3 \mathrm{mmol}), \mathrm{K}_{2} \mathrm{CO}_{3}$ aqueous solution $(2 \mathrm{M}, 10 \mathrm{~mL})$ and toluene $(30 \mathrm{~mL})$. The mixture was deoxygenated with nitrogen for $15 \mathrm{~min} . \mathrm{Pd}\left(\mathrm{PPh}_{3}\right)_{4}(173 \mathrm{mg}, 0.15 \mathrm{mmol})$ was added under nitrogen. The mixture was refluxed for $48 \mathrm{~h}$ and then cooled down to room temperature. Water (30 $\mathrm{mL})$ was added and the mixture was extracted with dichloromethane $(2 \times 20 \mathrm{~mL})$. The organic phase was dried over anhydrous $\mathrm{MgSO}_{4}$ and filtered. After removing the solvent from the filtrate, the residue was added into dichloromethane $(200 \mathrm{~mL})$ and filtered, yielding a yellow solid (674 $\mathrm{mg}$, crude yield: $82 \%$ ). The compound was directly used for next step reaction without further purification due to limited solubility. MS (MALDI-TOF): $\mathrm{m} / z$ 549.0 $\left(\mathrm{MH}^{+}\right)$.

NO. To a suspension of magnesium turnings (354 mg, $15 \mathrm{mmol})$ and a little iodine in dry THF (15 mL) was added 1-bromo-4-hexylbenzene (3.54 g, $15 \mathrm{mmol})$ dropwise under nitrogen, and then the mixture was stirred for $2 \mathrm{~h}$. To a solution of crude compound 5 (660 mg, ca. $1.2 \mathrm{mmol})$ in dry THF $(30 \mathrm{~mL})$ was added the prepared Grignard reagent dropwise at room temperature under nitrogen. The mixture was stirred at $80{ }^{\circ} \mathrm{C}$ for $12 \mathrm{~h}$ and then cooled down to room temperature. Saturated $\mathrm{NH}_{4} \mathrm{Cl}$ aqueous solution $(30 \mathrm{~mL})$ was added and the mixture was extracted with dichloromethane $(2$ $\times 30 \mathrm{~mL}$ ). The organic phase was dried over anhydrous $\mathrm{MgSO}_{4}$ and filtered. After removing the solvent, the brown residue was resolved by octane $(30 \mathrm{~mL})$ and HOAc $(5 \mathrm{~mL})$, then concentrated 
$\mathrm{H}_{2} \mathrm{SO}_{4}$ : $\mathrm{HOAc}(0.05: 1 \mathrm{~mL})$ was added dropwise, the mixture was stirred at reflux for $12 \mathrm{~h}$ and then quenched with water. The organic layer was washed with water for three times and extracted with dichloromethane $(2 \times 30 \mathrm{~mL})$, and was dried over anhydrous $\mathrm{MgSO}_{4}$ and filtered. After removing the solvent from the filtrate, the residue was purified by column chromatography on silica gel using petroleum ether/dichloromethane (20:1) as the eluent yielding a yellow solid (615 mg, two-step overall yield: $48 \%) .{ }^{1} \mathrm{H}$ NMR (300 MHz, $\left.\mathrm{CDCl}_{3}\right): \delta 7.79(\mathrm{~d}, J=8.4 \mathrm{~Hz}, 2 \mathrm{H}), 7.32(\mathrm{~d}, J=8.4 \mathrm{~Hz}, 2 \mathrm{H})$, $7.14(\mathrm{~d}, J=7.2 \mathrm{~Hz}, 8 \mathrm{H}), 7.07(\mathrm{~d}, J=4.5 \mathrm{~Hz}, 2 \mathrm{H}), 7.02(\mathrm{~d}, J=4.5 \mathrm{~Hz}, 2 \mathrm{H}), 6.91(\mathrm{~d}, J=7.2 \mathrm{~Hz}, 8 \mathrm{H})$, $2.40(\mathrm{t}, J=7.2 \mathrm{~Hz}, 8 \mathrm{H}), 1.43(\mathrm{~m}, 8 \mathrm{H}), 1.14(\mathrm{~m}, 24 \mathrm{H}), 0.73(\mathrm{~m}, 12 \mathrm{H}) .{ }^{13} \mathrm{C} \mathrm{NMR}\left(100 \mathrm{MHz}, \mathrm{CDCl}_{3}\right): \delta$ $149.70,149.32,143.02,141.74,141.68,137.65,134.94,133.38,129.00,128.41,126.42,125.92$, 120.39, 118.76, 64.49, 35.67, 31.79, 31.22, 29.27, 22.69, 14.18. MS (MALDI-TOF): $\mathrm{m} / z$ 1068.1 $\left(\mathrm{M}^{+}\right)$. Anal. calc. for $\mathrm{C}_{72} \mathrm{H}_{76} \mathrm{~S}_{4}: \mathrm{C}, 80.85 ; \mathrm{H}, 7.16$. Found: $\mathrm{C}, 80.94 ; \mathrm{H}, 7.01$.

NO-CHO. To a three-necked round bottom flask were added $\mathrm{POCl}_{3}(0.8 \mathrm{~mL})$ and DMF $(4 \mathrm{~mL})$ under the protection of nitrogen and the solution was stirred at $0{ }^{\circ} \mathrm{C}$ for $2 \mathrm{~h}$. Then, NO $(214 \mathrm{mg}, 0.2$ mmol) in 1,2-dichloroethane solution $(15 \mathrm{~mL})$ was added. After stirred at $80{ }^{\circ} \mathrm{C}$ for $12 \mathrm{~h}$, the mixture was quenched with saturated $\mathrm{CH}_{3} \mathrm{COONa}(\mathrm{aq})$ and extracted with dichloromethane $(2 \times 30 \mathrm{~mL})$. The organic phase was dried over anhydrous $\mathrm{MgSO}_{4}$ and filtered. After removing the solvent from the filtrate, the residue was purified by column chromatography on silica gel using petroleum ether/dichloromethane (3:1) as the eluent yielding an orange solid (209 mg, 93\%). ${ }^{1} \mathrm{H}$ NMR (300 $\left.\mathrm{MHz}, \mathrm{CD}_{2} \mathrm{Cl}_{2}\right): \delta 9.82(\mathrm{~s}, 2 \mathrm{H}), 7.96(\mathrm{~d}, J=8.7 \mathrm{~Hz}, 2 \mathrm{H}), 7.92(\mathrm{~s}, 2 \mathrm{H}), 7.59(\mathrm{~d}, J=8.7 \mathrm{~Hz}, 2 \mathrm{H}), 7.21$ $(\mathrm{d}, J=7.8 \mathrm{~Hz}, 2 \mathrm{H}), 7.07(\mathrm{~d}, J=7.8 \mathrm{~Hz}, 2 \mathrm{H}), 2.51(\mathrm{t}, J=7.5 \mathrm{~Hz}, 8 \mathrm{H}), 1.52(\mathrm{~m}, 8 \mathrm{H}), 1.25(\mathrm{~m}, 24 \mathrm{H})$, $0.81(\mathrm{~m}, 12 \mathrm{H}) .{ }^{13} \mathrm{C} \mathrm{NMR}\left(100 \mathrm{MHz}, \mathrm{CD}_{2} \mathrm{Cl}_{2}\right): \delta 182.87,150.96,149.77,149.50,143.70,142.26$, $141.65,139.62,136.50,134.64,129.99,129.50,129.24,128.75,128.67,127.04,119.39,64.53$, 
35.60, 31.73, 31.22, 29.21, 22.64, 14.16. MS (MALDI-TOF): $m / z 1125.1\left(\mathrm{M}^{+}\right)$. Anal. calc. for $\mathrm{C}_{74} \mathrm{H}_{76} \mathrm{O}_{2} \mathrm{~S}_{4}:$ C, 78.96; H, 6.81. Found: C, 78.91; H, 6.88.

NOIC. To a three-necked round bottom flask were added NO-CHO (225 mg, $0.2 \mathrm{mmol}), 2 \mathrm{FIC}$ (184 mg, $0.8 \mathrm{mmol})$, pyridine $(0.5 \mathrm{~mL})$ and chloroform $\left(\mathrm{CHCl}_{3}\right)(30 \mathrm{~mL})$. The mixture was deoxygenated with nitrogen for $15 \mathrm{~min}$ and then stirred at reflux for $12 \mathrm{~h}$. After cooling down to room temperature, the mixture was poured into methanol $(150 \mathrm{~mL})$ and filtered. The residue was purified by column chromatography on silica gel using petroleum ether/dichloromethane $(2: 1)$ as eluent yielding a purple solid (236 mg, 76\%). ${ }^{1} \mathrm{H}$ NMR (300 MHz, $\left.\mathrm{CDCl}_{3}\right): \delta 8.78(\mathrm{~s}, 2 \mathrm{H}), 8.48(\mathrm{~m}$, 2H), $8.14(\mathrm{~s}, 2 \mathrm{H}), 8.01(\mathrm{~d}, J=9.0 \mathrm{~Hz}, 2 \mathrm{H}), 7.64(\mathrm{~m}, 4 \mathrm{H}), 7.28(\mathrm{~d}, J=6.6 \mathrm{~Hz}, 8 \mathrm{H}), 7.12(\mathrm{~d}, J=6.6$ $\mathrm{Hz}, 8 \mathrm{H}), 2.53(\mathrm{~m}, 8 \mathrm{H}), 1.54(\mathrm{~m}, 8 \mathrm{H}), 1.24(\mathrm{~m}, 24 \mathrm{H}), 0.81(\mathrm{~m}, 12 \mathrm{H}) .{ }^{13} \mathrm{C} \mathrm{NMR}\left(75 \mathrm{MHz}, \mathrm{CDCl}_{3}\right): \delta$ $185.87,154.07,152.45,150.59,147.38,143.84,142.60,138.84,137.72,136.74,136.13,134.90$, $134.59,129.94,128.91,128.84,127.67,121.48,119.78,115.21,114.92,114.40,114.27,112.89$, $112.65,69.68,64.73,35.67,31.79,31.25,29.28,22.68,14.19$. MS (MALDI-TOF): $\mathrm{m} / z$ 1549.3 $\left(\mathrm{MH}^{+}\right)$. Anal. calc. for $\mathrm{C}_{98} \mathrm{H}_{80} \mathrm{~F}_{4} \mathrm{~N}_{4} \mathrm{O}_{2} \mathrm{~S}_{4}: \mathrm{C}, 75.94 ; \mathrm{H}, 5.20 ; \mathrm{N}, 3.61$. Found: $\mathrm{C}, 75.88 ; \mathrm{H}, 5.23 ; \mathrm{N}$, 3.67 .
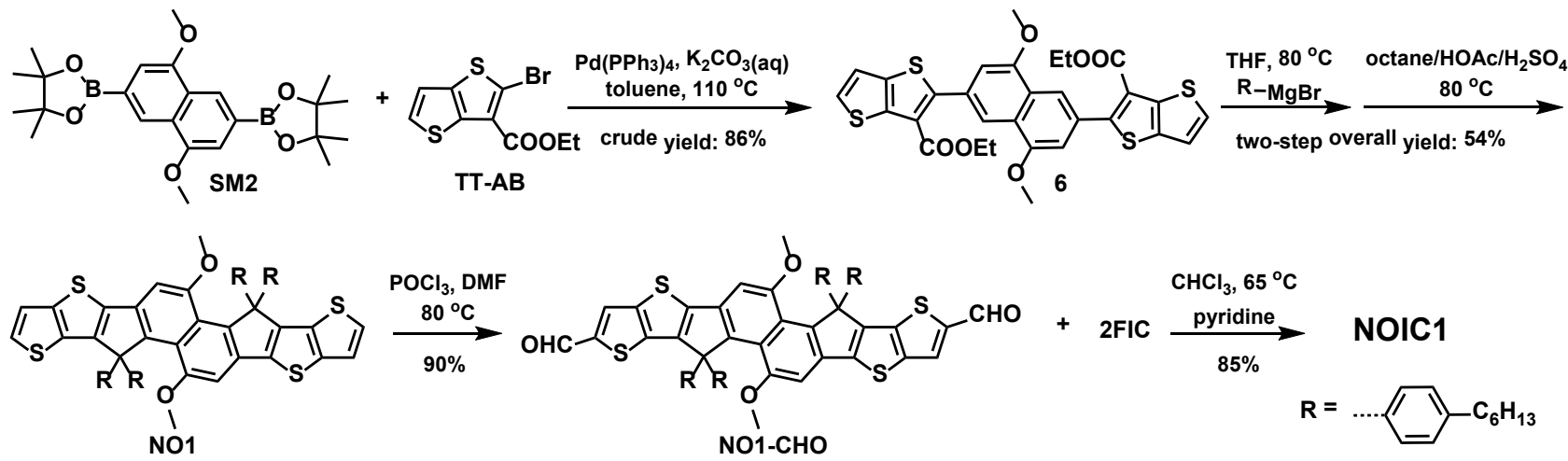

Scheme S3. Synthetic route for NOIC1

Compound 6. This compound was prepared following the same procedure as for compound 5. 
the crude product was afforded as a yellow solid (784 mg, crude yield: $86 \%)$. The compound was directly used for next step reaction without further purification due to limited solubility. MS (MALDI-TOF): $m / z 608.0\left(\mathrm{M}^{+}\right)$.

NO1. This compound was prepared following the same procedure as for NO. The product was afforded as a yellow solid (732 mg, two-step overall yield: 54\%). ${ }^{1} \mathrm{H}$ NMR $\left(300 \mathrm{MHz}, \mathrm{CDCl}_{3}\right): \delta$ $7.40(\mathrm{~d}, J=7.8 \mathrm{~Hz}, 8 \mathrm{H}), 7.20(\mathrm{~m}, 4 \mathrm{H}), 7.06(\mathrm{~d}, J=7.8 \mathrm{~Hz}, 8 \mathrm{H}), 6.87(\mathrm{~s}, 2 \mathrm{H}), 3.32(\mathrm{~m}, 6 \mathrm{H}), 2.58(\mathrm{t}, J$ $=7.2 \mathrm{~Hz}, 8 \mathrm{H}), 1.61(\mathrm{~m}, 8 \mathrm{H}), 1.32(\mathrm{~m}, 24 \mathrm{H}), 0.91(\mathrm{~m}, 12 \mathrm{H}) .{ }^{13} \mathrm{C} \mathrm{NMR}\left(75 \mathrm{MHz}, \mathrm{CDCl}_{3}\right): \delta 156.12$, $151.54,141.40,140.97,138.20,137.02,133.57,128.05,127.49,125.73,124.71,120.04,66.88$, 53.67, 35.53, 31.78, 31.28, 29.08, 22.68, 14.16. MS (MALDI-TOF): $m / z$ 1129.4 $\left(\mathrm{MH}^{+}\right)$. Anal. calc. for $\mathrm{C}_{74} \mathrm{H}_{80} \mathrm{O}_{2} \mathrm{~S}_{4}: \mathrm{C}, 78.68 ; \mathrm{H}, 7.14$. Found: $\mathrm{C}, 78.62 ; \mathrm{H}, 7.18$.

NO1-CHO. This compound was prepared following the same procedure as for NO-CHO. The product was afforded as an orange solid (213 mg, 90\%). ${ }^{1} \mathrm{H}$ NMR (300 MHz, $\left.\mathrm{CDCl}_{3}\right): \delta 9.79(\mathrm{~s}, 2 \mathrm{H})$, $7.80(\mathrm{~s}, 2 \mathrm{H}), 7.32(\mathrm{~d}, J=7.2 \mathrm{~Hz}, 8 \mathrm{H}), 7.03(\mathrm{~d}, J=7.2 \mathrm{~Hz}, 8 \mathrm{H}), 6.86(\mathrm{~s}, 2 \mathrm{H}), 3.30(\mathrm{~s}, 6 \mathrm{H}), 2.53(\mathrm{t}, J=$ $7.2 \mathrm{~Hz}, 8 \mathrm{H}), 1.56(\mathrm{~m}, 8 \mathrm{H}), 1.27(\mathrm{~m}, 24 \mathrm{H}), 0.85(\mathrm{~m}, 12 \mathrm{H}) .{ }^{13} \mathrm{C} \mathrm{NMR}\left(75 \mathrm{MHz}, \mathrm{CDCl}_{3}\right): \delta 182.93$, $156.47,151.52,147.87,143.42,142.72,141.42,140.90,140.21,137.11,136.90,129.82,127.86$ 127.82, 125.46, 100.26, 67.13, 53.98, 35.57, 31.81, 31.32, 29.15, 22.72, 14.20. MS (MALDI-TOF): $m / z$ 1184.4 (M $\left.\mathrm{M}^{+}\right)$. Anal. calc. for $\mathrm{C}_{76} \mathrm{H}_{80} \mathrm{O}_{4} \mathrm{~S}_{4}$ : C, 76.99; H, 6.80. Found: C, 77.05; H, 6.75.

NOIC1. This compound was prepared following the same procedure as for NOIC. The product was afforded as a purple solid $(274 \mathrm{mg}, 85 \%) .{ }^{1} \mathrm{H}$ NMR $\left(300 \mathrm{MHz}, \mathrm{CDCl}_{3}\right): \delta 8.70(\mathrm{~s}, 2 \mathrm{H}), 8.46(\mathrm{~m}$, 2H), 7.98 (s, 2H), $7.72(\mathrm{~m}, 2 \mathrm{H}), 7.40$ (d, $J=7.2 \mathrm{~Hz}, 8 \mathrm{H}), 7.12(\mathrm{~d}, J=7.2 \mathrm{~Hz}, 8 \mathrm{H}), 6.85$ (s, 2H), 3.31 $(\mathrm{s}, 6 \mathrm{H}), 2.57(\mathrm{~m}, 8 \mathrm{H}), 1.59(\mathrm{~m}, 8 \mathrm{H}), 1.27(\mathrm{~m}, 24 \mathrm{H}), 0.83(\mathrm{~m}, 12 \mathrm{H}) .{ }^{13} \mathrm{C} \mathrm{NMR}\left(75 \mathrm{MHz}, \mathrm{CDCl}_{3}\right): \delta$ $185.63,158.19,156.94,156.28,156.10,152.79,152.62,147.61,144.59,143.88,141.19,138.29$, 
$137.35,136.57,134.48,128.48,128.30,128.02,127.91,125.62,121.14,115.00,114.71,114.48$, $114.31,112.79,112.54,100.32,69.19,67.16,54.18,35.55,31.80,31.28,29.12,22.70,14.17 . \mathrm{MS}$ (MALDI-TOF): $m / z$ 1609.4 $\left(\mathrm{M}^{+}\right)$. Anal. calc. for $\mathrm{C}_{100} \mathrm{H}_{84} \mathrm{~F}_{4} \mathrm{~N}_{4} \mathrm{O}_{4} \mathrm{~S}_{4}: \mathrm{C}, 74.60 ; \mathrm{H}, 5.26 ; \mathrm{N}, 3.48$. Found: C, 74.67; H, 5.23; N, 3.46.
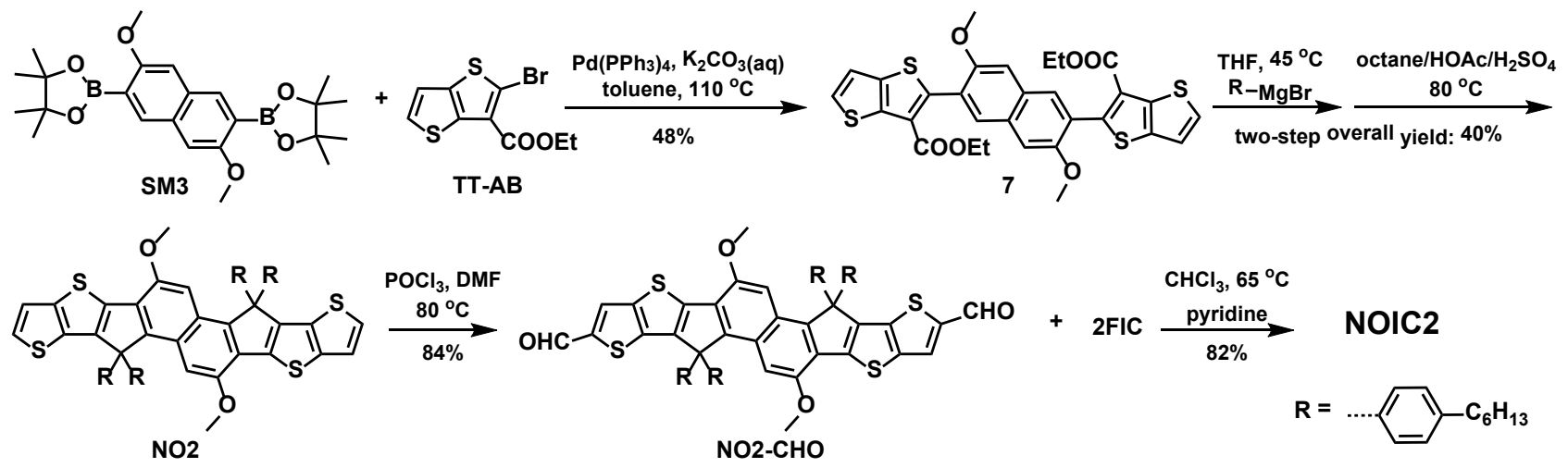

Scheme S4. Synthetic route for NOIC2

Compound 7. To a three-necked round bottom flask were added SM3 (660 mg, $1.5 \mathrm{mmol}$ ), TT-AB (873 mg, $3 \mathrm{mmol}), \mathrm{K}_{2} \mathrm{CO}_{3}$ aqueous solution $(2 \mathrm{M}, 10 \mathrm{~mL})$ and toluene (30 mL). The mixture was deoxygenated with nitrogen for $15 \mathrm{~min} . \mathrm{Pd}\left(\mathrm{PPh}_{3}\right)_{4}(173 \mathrm{mg}, 0.15 \mathrm{mmol})$ was added under nitrogen. The mixture was refluxed for $48 \mathrm{~h}$ and then cooled down to room temperature. Water (30 $\mathrm{mL}$ ) was added and the mixture was extracted with dichloromethane $(2 \times 20 \mathrm{~mL})$. The organic phase was filtered. After removing the solvent from the filtrate, the residue was purified by column chromatography on silica gel using petroleum ether/dichloromethane (1:2) as eluent yielding a yellow solid (438 mg, 48\%). ${ }^{1} \mathrm{H}$ NMR (300 MHz, $\left.\mathrm{CD}_{2} \mathrm{Cl}_{2}\right): \delta 7.79(\mathrm{~s}, 2 \mathrm{H}), 7.48(\mathrm{~d}, J=5.1 \mathrm{~Hz}, 2 \mathrm{H})$, $7.30(\mathrm{~d}, J=5.1 \mathrm{~Hz}, 2 \mathrm{H}), 7.19(\mathrm{~m}, 2 \mathrm{H}), 4.18(\mathrm{q}, J=7.2 \mathrm{~Hz}, 4 \mathrm{H}), 3.84(\mathrm{~s}, 6 \mathrm{H}), 1.11(\mathrm{t}, J=6.8 \mathrm{~Hz}, 6 \mathrm{H})$. ${ }^{13} \mathrm{C}$ NMR $\left(75 \mathrm{MHz}, \mathrm{CD}_{2} \mathrm{Cl}_{2}\right): \delta 162.21,154.25,147.18,140.06,136.60,129.28,129.25,128.41$, 126.09, 123.43, 119.00, 105.74, 60.75, 55.62, 13.86. MS (MALDI-TOF): $m / z 608.1\left(\mathrm{M}^{+}\right)$. Anal. calc. for $\mathrm{C}_{30} \mathrm{H}_{24} \mathrm{O}_{6} \mathrm{~S}_{4}: \mathrm{C}, 59.19 ; \mathrm{H}, 3.97$. Found: $\mathrm{C}, 59.25 ; \mathrm{H}, 3.91$. 
NO2. To a suspension of magnesium turnings $(374 \mathrm{mg}, 16 \mathrm{mmol})$ and a little iodine in dry THF $(15 \mathrm{~mL})$ was added 1-bromo-4-hexylbenzene $(3.76 \mathrm{~g}, 16 \mathrm{mmol})$ dropwise under nitrogen, and then the mixture was stirred for $2 \mathrm{~h}$. To a solution of crude compound 7 (790 mg, ca. $1.3 \mathrm{mmol}$ ) in dry THF $(30 \mathrm{~mL})$ was added the prepared Grignard reagent dropwise at room temperature under nitrogen. The mixture was stirred at $45{ }^{\circ} \mathrm{C}$ for $48 \mathrm{~h}$ and then cooled down to room temperature. Saturated $\mathrm{NH}_{4} \mathrm{Cl}$ aqueous solution $(30 \mathrm{~mL})$ was added and the mixture was extracted with dichloromethane $(2$ $\times 30 \mathrm{~mL}$ ). The organic phase was dried over anhydrous $\mathrm{MgSO}_{4}$ and filtered. After removing the solvent, the brown residue was resolved by octane $(30 \mathrm{~mL})$ and HOAc $(5 \mathrm{~mL})$, then concentrated $\mathrm{H}_{2} \mathrm{SO}_{4}$ : HOAc $(0.06: 1 \mathrm{~mL})$ was added dropwise, the mixture was stirred at reflux for $12 \mathrm{~h}$ and then quenched with water. The organic layer was washed with water for three times and extracted with dichloromethane $(2 \times 30 \mathrm{~mL})$, and was dried over anhydrous $\mathrm{MgSO}_{4}$ and filtered. After removing the solvent from the filtrate, the residue was purified by column chromatography on silica gel using petroleum ether/dichloromethane (30:1) as the eluent yielding a yellow solid (588 mg, two-step overall yield: 40\%). ${ }^{1} \mathrm{H}$ NMR (400 MHz, $\left.\mathrm{CDCl}_{3}\right): \delta 7.25(\mathrm{~m}, 10 \mathrm{H}), 7.21(\mathrm{~m}, 2 \mathrm{H}), 7.14(\mathrm{~s}, 2 \mathrm{H}), 7.05$ $(\mathrm{d}, J=8.0 \mathrm{~Hz}, 8 \mathrm{H}), 3.76(\mathrm{~s}, 6 \mathrm{H}), 2.55(\mathrm{t}, J=8.0 \mathrm{~Hz}, 8 \mathrm{H}), 1.56(\mathrm{~m}, 8 \mathrm{H}), 1.27(\mathrm{~m}, 24 \mathrm{H}), 0.86(\mathrm{~m}$, 12H). ${ }^{13} \mathrm{C} \mathrm{NMR}\left(75 \mathrm{MHz}, \mathrm{CDCl}_{3}\right): \delta 149.46,149.18,148.61,143.32,141.57,140.39,137.27,132.99$, $128.98,128.23,127.72,126.10,125.28,120.34,104.13,64.61,55.41,35.52,31.68,31.11,29.06$, 22.56, 14.03. MS (MALDI-TOF): $m / z 1129.3\left(\mathrm{MH}^{+}\right)$. Anal. calc. for $\mathrm{C}_{74} \mathrm{H}_{80} \mathrm{O}_{2} \mathrm{~S}_{4}: \mathrm{C}, 78.68 ; \mathrm{H}, 7.14$. Found: C, 78.74; H, 7.10.

NO2-CHO. This compound was prepared following the same procedure as for NO-CHO. The product was afforded as an orange solid (199 mg, 84\%). ${ }^{1} \mathrm{H}$ NMR (400 MHz, $\left.\mathrm{CDCl}_{3}\right): \delta 9.86(\mathrm{~s}, 2 \mathrm{H})$, $7.91(\mathrm{~s}, 2 \mathrm{H}), 7.21(\mathrm{~d}, J=8.4 \mathrm{~Hz}, 8 \mathrm{H}), 7.16(\mathrm{~s}, 2 \mathrm{H}), 7.07(\mathrm{~d}, J=8.4 \mathrm{~Hz}, 8 \mathrm{H}), 3.76(\mathrm{~s}, 6 \mathrm{H}), 2.54(\mathrm{t}, J=$ 
$8.0 \mathrm{~Hz}, 8 \mathrm{H}), 1.56(\mathrm{~m}, 8 \mathrm{H}), 1.27(\mathrm{~m}, 24 \mathrm{H}), 0.85(\mathrm{~m}, 12 \mathrm{H}) .{ }^{13} \mathrm{C} \mathrm{NMR}\left(100 \mathrm{MHz}, \mathrm{CDCl}_{3}\right): \delta 182.97$, $150.89,149.96,148.89,147.29,143.38,143.35,142.29,139.51,136.31,130.12,128.89,128.63$, 127.80, 126.83, 104.67, 64.82, 55.69, 35.62, 31.77, 31.22, 29.17, 22.67, 14.14. MS (MALDI-TOF): $m / z$ 1184.6 $\left(\mathrm{M}^{+}\right)$. Anal. calc. for $\mathrm{C}_{76} \mathrm{H}_{80} \mathrm{O}_{4} \mathrm{~S}_{4}: \mathrm{C}, 76.99 ; \mathrm{H}, 6.80$. Found: C, 77.90; H, 6.85 .

NOIC2. This compound was prepared following the same procedure as for NOIC. The product was afforded as a dark solid (264 mg, 82\%). ${ }^{1} \mathrm{H}$ NMR (400 MHz, $\left.\mathrm{CDCl}_{3}\right): \delta 8.79(\mathrm{~s}, 2 \mathrm{H}), 8.48(\mathrm{~m}$, 2H), $8.06(\mathrm{~s}, 2 \mathrm{H}), 7.69(\mathrm{~m}, 2 \mathrm{H}), 7.28(\mathrm{~d}, J=8.0 \mathrm{~Hz}, 8 \mathrm{H}), 7.20(\mathrm{~s}, 2 \mathrm{H}), 7.13(\mathrm{~d}, J=8.0 \mathrm{~Hz}, 8 \mathrm{H}), 3.74$ (s, 6H), $2.56(\mathrm{~m}, 8 \mathrm{H}), 1.58(\mathrm{~m}, 8 \mathrm{H}), 1.27(\mathrm{~m}, 24 \mathrm{H}), 0.83(\mathrm{~m}, 12 \mathrm{H}) .{ }^{13} \mathrm{C} \mathrm{NMR}\left(100 \mathrm{MHz}, \mathrm{CDCl}_{3}\right): \delta$ $185.86,158.34,155.85,155.72,153.25,153.12,152.18,150.26,149.57,147.35,145.66,142.59$, $138.62,138.52,138.07,136.64,135.86,134.54,128.92,128.83,127.34,121.04,115.06,114.85$, $114.47,114.35,112.72,112.54,104.97,69.28,64.96,55.80,35.64,31.77,31.21,29.18,22.66,14.13$. MS (MALDI-TOF): $m / z$ 1609.5 $\left(\mathrm{M}^{+}\right)$. Anal. calc. for $\mathrm{C}_{100} \mathrm{H}_{84} \mathrm{~F}_{4} \mathrm{~N}_{4} \mathrm{O}_{4} \mathrm{~S}_{4}: \mathrm{C}, 74.60 ; \mathrm{H}, 5.26 ; \mathrm{N}, 3.48$. Found: C, 74.51; H, 5.33; N, 3.56.
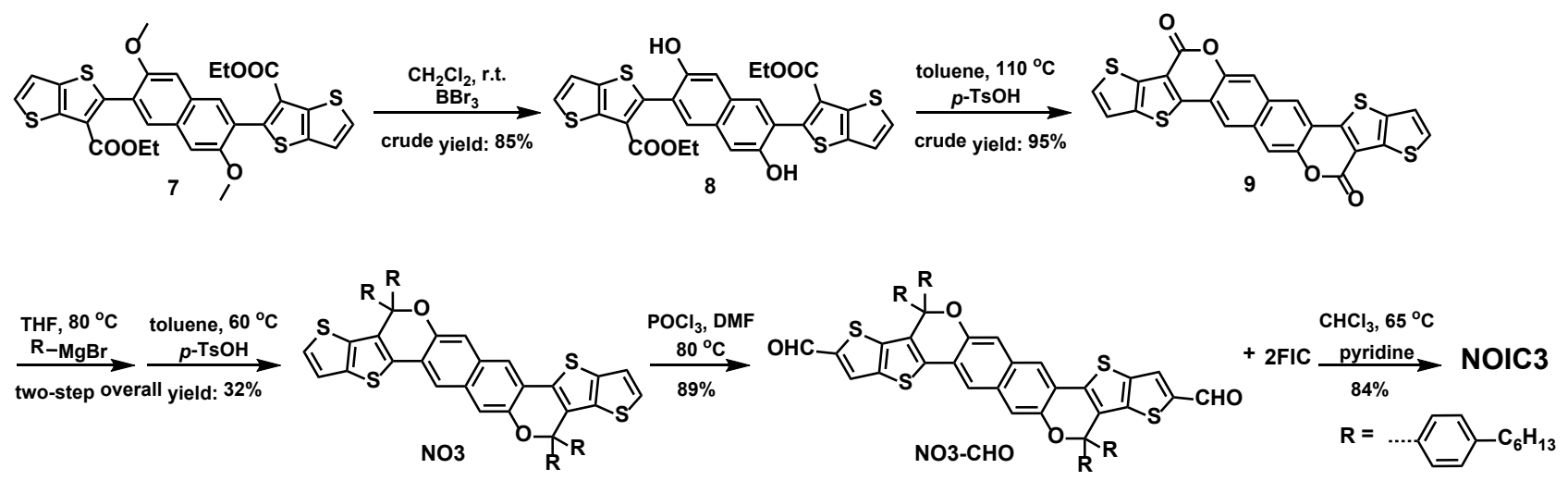

Scheme S5. Synthetic route for NOIC3.

Compound 8. To a solution of compound $7(608 \mathrm{mg}, 1 \mathrm{mmol})$ in dry dichloromethane $(30 \mathrm{~mL})$ at $-78{ }^{\circ} \mathrm{C}$ was added $1 \mathrm{M} \mathrm{BBr}_{3}$ in dry dichloromethane $(6 \mathrm{~mL}, 6 \mathrm{mmol})$ dropwise under nitrogen. The mixture was stirred at $-78{ }^{\circ} \mathrm{C}$ for $0.5 \mathrm{~h}$ and stirred overnight at room temperature. Water $(30 \mathrm{~mL})$ 
was added and the mixture was extracted with dichloromethane $(2 \times 20 \mathrm{~mL})$. The organic phase was dried over anhydrous $\mathrm{MgSO}_{4}$ and filtered. After removing the solvent from the filtrate, the residue was added into dichloromethane $(100 \mathrm{~mL})$ and filtered, yielding a yellow solid (493 mg, crude yield: $85 \%$ ). The compound was directly used for next step reaction without further purification due to limited solubility. MS (MALDI-TOF): $m / z 579.9\left(\mathrm{M}^{+}\right)$.

Compound 9. To a three-necked round bottom flask were added crude compound 8 (580 mg, $c a$. $1 \mathrm{mmol}), p$-toluenesulfonic acid $(200 \mathrm{mg}, 1.05 \mathrm{mmol})$ and toluene $(30 \mathrm{~mL})$. The mixture was deoxygenated with nitrogen for $15 \mathrm{~min}$. The mixture was refluxed for $12 \mathrm{~h}$ and then cooled down to room temperature. The mixture was poured into methanol $(100 \mathrm{~mL})$ and filtered, yielding a yellow solid (463 mg, crude yield: 95\%). The compound was directly used for next step reaction without further purification due to limited solubility. MS (MALDI-TOF): $m / z 488.0\left(\mathrm{M}^{+}\right)$.

NO3. To a suspension of magnesium turnings $(248 \mathrm{mg}, 10 \mathrm{mmol})$ and a little iodine in dry THF (15 mL) was added 1-bromo-4-hexylbenzene (2.49 g, $10 \mathrm{mmol})$ dropwise under nitrogen, and then the mixture was stirred for $2 \mathrm{~h}$. To a solution of compound $9(420 \mathrm{mg}, c a .0 .86 \mathrm{mmol})$ in dry THF $(20 \mathrm{~mL})$ was added the prepared Grignard reagent dropwise at room temperature under nitrogen. The mixture was stirred at reflux for $12 \mathrm{~h}$ and then cooled down to room temperature. Saturated $\mathrm{NH}_{4} \mathrm{Cl}$ aqueous solution $(30 \mathrm{~mL})$ was added and the mixture was extracted with dichloromethane $(2 \times 30$ $\mathrm{mL}$ ). The organic phase was dried over anhydrous $\mathrm{MgSO}_{4}$ and filtered. After removing the solvent, the brown residue was dissolved in toluene $(30 \mathrm{~mL})$, then $p$-toluenesulfonic acid $(380 \mathrm{mg}, 2 \mathrm{mmol})$ was added, the mixture was stirred at $60{ }^{\circ} \mathrm{C}$ for $12 \mathrm{~h}$ and then cooled down to room temperature. Water $(30 \mathrm{~mL})$ was added and the mixture was extracted with dichloromethane $(2 \times 30 \mathrm{~mL})$. The organic phase was dried over anhydrous $\mathrm{MgSO}_{4}$ and filtered. After removing the solvent from the 
filtrate, the residue was purified by column chromatography on silica gel using petroleum ether/dichloromethane (10:1) as the eluent yielding a yellow solid (303 mg, two-step overall yield: 32\%). ${ }^{1} \mathrm{H}$ NMR (400 MHz, $\left.\mathrm{CD}_{2} \mathrm{Cl}_{2}\right): \delta 7.56(\mathrm{~s}, 2 \mathrm{H}), 7.28(\mathrm{~s}, 2 \mathrm{H}), 7.23(\mathrm{~d}, J=8.0 \mathrm{~Hz}, 8 \mathrm{H}), 7.21(\mathrm{~m}$, 4H), $7.11(\mathrm{~d}, J=8.0 \mathrm{~Hz}, 8 \mathrm{H}), 2.56(\mathrm{t}, J=8.0 \mathrm{~Hz}, 8 \mathrm{H}), 1.58(\mathrm{~m}, 8 \mathrm{H}), 1.29(\mathrm{~m}, 24 \mathrm{H}), 0.87(\mathrm{~m}, 12 \mathrm{H})$. ${ }^{13} \mathrm{C}$ NMR $\left(100 \mathrm{MHz}, \mathrm{CD}_{2} \mathrm{Cl}_{2}\right): \delta 149.03,143.43,139.64,139.51,137.64,134.76,133.04,130.36$, $128.63,128.49,128.11,122.36,119.85,119.43,113.10,85.72,35.59,31.74,31.35,29.02,22.65$, 13.89. MS (MALDI-TOF): $m / z 1101.5\left(\mathrm{MH}^{+}\right)$. Anal. calc. for $\mathrm{C}_{72} \mathrm{H}_{76} \mathrm{O}_{2} \mathrm{~S}_{4}: \mathrm{C}, 78.50 ; \mathrm{H}, 6.95$. Found: C, 78.42; H, 7.02 .

NO3-CHO. This compound was prepared following the same procedure as for NO-CHO. The product was afforded as an orange solid (206 mg, 89\%). ${ }^{1} \mathrm{H} \mathrm{NMR}\left(400 \mathrm{MHz}, \mathrm{CD}_{2} \mathrm{Cl}_{2}\right): \delta 9.76(\mathrm{~s}, 2 \mathrm{H})$, $7.75(\mathrm{~s}, 2 \mathrm{H}), 7.50(\mathrm{~s}, 2 \mathrm{H}), 7.23(\mathrm{~m}, 10 \mathrm{H}), 7.13(\mathrm{~d}, J=8.0 \mathrm{~Hz}, 8 \mathrm{H}), 2.58(\mathrm{t}, J=8.0 \mathrm{~Hz}, 8 \mathrm{H}), 1.57(\mathrm{~m}$, 8H), $1.27(\mathrm{~m}, 24 \mathrm{H}), 0.85(\mathrm{~m}, 12 \mathrm{H}) .{ }^{13} \mathrm{C}$ NMR $\left(75 \mathrm{MHz}, \mathrm{CDCl}_{3}\right): \delta 182.90,149.05,145.69,143.84$, $140.72,138.83,137.36,133.16,130.85,128.77,128.39,128.30,125.50,121.99,121.19,113.62$, 85.60, 35.56, 31.69, 31.25, 29.01, 22.60, 13.86. MS (MALDI-TOF): $m / z 1157.3\left(\mathrm{M}^{+}\right)$. Anal. calc. for $\mathrm{C}_{74} \mathrm{H}_{76} \mathrm{O}_{4} \mathrm{~S}_{4}:$ C, 76.78; H, 6.62. Found: C, 76.88; H, 6.59.

NOIC3. This compound was prepared following the same procedure as for NOIC. The product was afforded as a purple solid (266 mg, 84\%). ${ }^{1} \mathrm{H}$ NMR (400 MHz, $\left.\mathrm{CDCl}_{3}\right): \delta 8.62(\mathrm{~s}, 2 \mathrm{H}), 8.41(\mathrm{~m}$, 2H), $8.00(\mathrm{~s}, 2 \mathrm{H}), 7.60(\mathrm{~m}, 2 \mathrm{H}), 7.41(\mathrm{~s}, 2 \mathrm{H}), 7.27(\mathrm{~d}, J=8.8 \mathrm{~Hz}, 8 \mathrm{H}), 7.18(\mathrm{~m}, 10 \mathrm{H}), 2.60(\mathrm{t}, J=8.0$ $\mathrm{Hz}, 8 \mathrm{H}), 1.62(\mathrm{~m}, 8 \mathrm{H}), 1.32(\mathrm{~m}, 8 \mathrm{H}), 1.26(\mathrm{~m}, 16 \mathrm{H}), 0.83(\mathrm{~m}, 12 \mathrm{H}) .{ }^{13} \mathrm{C}$ NMR $\left(100 \mathrm{MHz}, \mathrm{CDCl}_{3}\right): \delta$ $185.07,158.10,155.95,155.89,155.75,153.32,153.30,153.19,149.30,143.92,140.38,138.76$, $137.98,136.54,136.48,135.70,134.57,133.67,131.41,128.58,128.43,122.61,122.20,121.99$, $115.05,114.83,114.17,114.02,113.99,112.77,112.59,85.71,70.44,35.80,31.84,31.36,29.16$ 
22.68, 14.14. MS (MALDI-TOF): $m / z$ 1582.2 $\left(\mathrm{M}^{+}\right)$. Anal. calc. for $\mathrm{C}_{98} \mathrm{H}_{80} \mathrm{~F}_{4} \mathrm{~N}_{4} \mathrm{O}_{4} \mathrm{~S}_{4}:$ C, 74.41; $\mathrm{H}$, 5.10; N, 3.54. Found: C, 74.49; H, 5.03; N, 3.57.
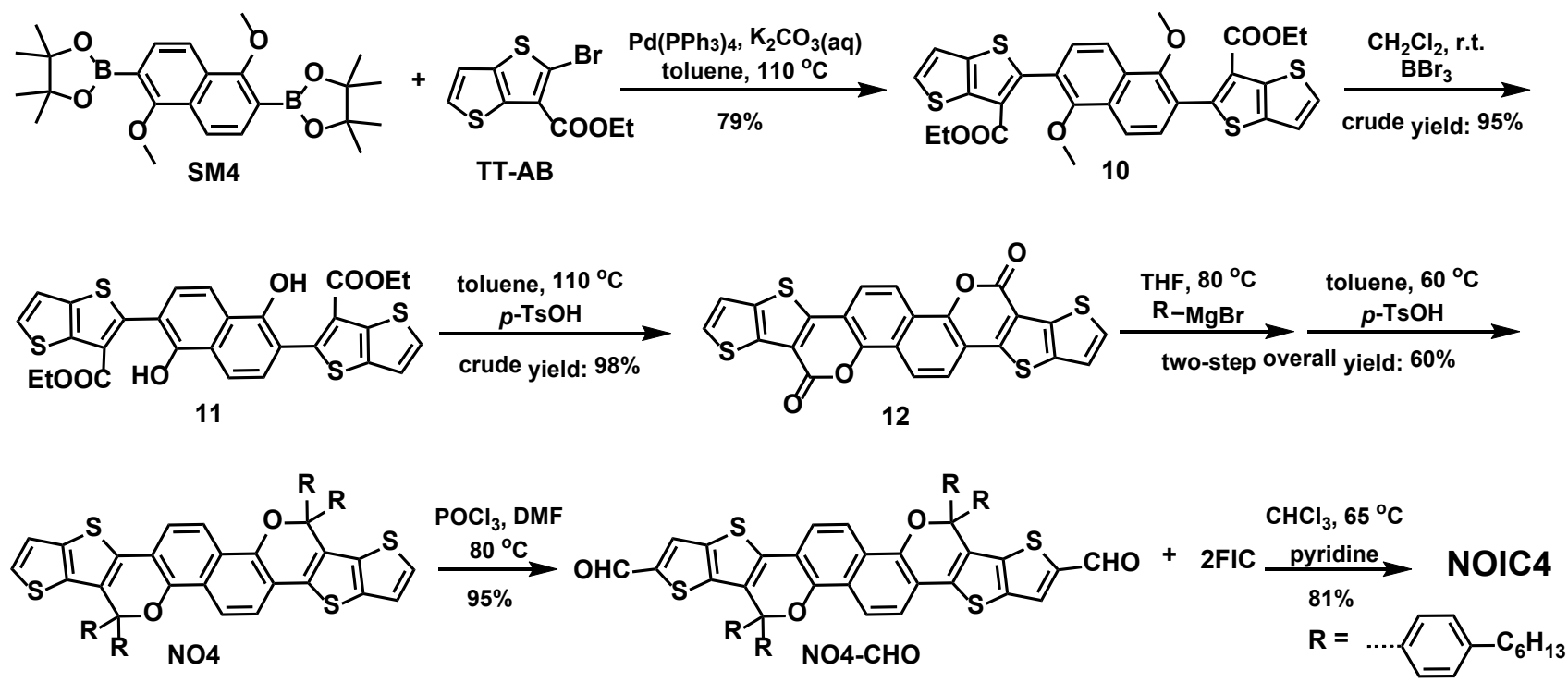

Scheme S6. Synthetic route for NOIC4

Compound 10. This compound was prepared following the same procedure as for compound 5.

The product was afforded as a pale yellow solid (720 mg, 79\%). ${ }^{1} \mathrm{H}$ NMR (400 $\left.\mathrm{MHz}, \mathrm{CD}_{2} \mathrm{Cl}_{2}\right): \delta$ $7.99(\mathrm{~d}, J=8.4 \mathrm{~Hz}, 2 \mathrm{H}), 7.53(\mathrm{~m}, 4 \mathrm{H}), 7.33(\mathrm{~d}, J=5.2 \mathrm{~Hz}, 2 \mathrm{H}), 4.20(\mathrm{q}, J=7.2 \mathrm{~Hz}, 4 \mathrm{H}), 3.71(\mathrm{~m}$, $6 \mathrm{H}), 1.09(\mathrm{t}, J=6.8 \mathrm{~Hz}, 6 \mathrm{H}) .{ }^{13} \mathrm{C} \mathrm{NMR}\left(100 \mathrm{MHz}, \mathrm{CD}_{2} \mathrm{Cl}_{2}\right): \delta 162.53,154.94,148.24,140.32$, $137.73,130.14,129.93,129.15,124.27,123.39,119.44,118.30,62.30,61.27,30.11,14.15 . \mathrm{MS}$ (MALDI-TOF): $m / z$ 608.1 (M $\mathrm{M}^{+}$). Anal. calc. for $\mathrm{C}_{30} \mathrm{H}_{24} \mathrm{O}_{6} \mathrm{~S}_{4}: \mathrm{C}, 59.19 ; \mathrm{H}, 3.97$. Found: $\mathrm{C}, 59.27$; $\mathrm{H}$, 3.93.

Compound 11. This compound was prepared following the same procedure as for compound 8 . The product was afforded as a pale yellow solid (551 mg, crude yield: 95\%). The compound was directly used for next step reaction without further purification due to limited solubility. MS (MALDI-TOF): $m / z 580.0\left(\mathrm{M}^{+}\right)$.

Compound 12. This compound was prepared following the same procedure as for compound 9. 
The product was afforded as a yellow solid (478 mg, crude yield: 98\%). The compound was directly used for next step reaction without further purification due to limited solubility. MS (MALDI-TOF): $m / z 487.9\left(\mathrm{M}^{+}\right)$.

NO4. This compound was prepared following the same procedure as for NO3. The product was afforded as a yellow solid (568 mg, two-step overall yield: $60 \%) .{ }^{1} \mathrm{H}$ NMR (400 $\left.\mathrm{MHz}, \mathrm{CDCl}_{3}\right): \delta$ $7.96(\mathrm{~d}, J=8.4 \mathrm{~Hz}, 2 \mathrm{H}), 7.39(\mathrm{~d}, J=8.4 \mathrm{~Hz}, 2 \mathrm{H}), 7.28(\mathrm{~d}, J=8.0 \mathrm{~Hz}, 8 \mathrm{H}), 7.22(\mathrm{~d}, J=5.2 \mathrm{~Hz}, 8 \mathrm{H})$, $7.15(\mathrm{~d}, J=5.2 \mathrm{~Hz}, 2 \mathrm{H}), 7.08(\mathrm{~d}, J=8.0 \mathrm{~Hz}, 8 \mathrm{H}), 2.56(\mathrm{t}, J=8.0 \mathrm{~Hz}, 8 \mathrm{H}), 1.58(\mathrm{~m}, 8 \mathrm{H}), 1.28(\mathrm{~m}$, 24H), $0.86(\mathrm{~m}, 12 \mathrm{H}) .{ }^{13} \mathrm{C}$ NMR (100 MHz, $\left.\mathrm{CDCl}_{3}\right): \delta 147.92,146.90,143.23,140.09,139.77$, $137.11,135.41,128.81,128.39,128.17,125.95,121.00,119.46,116.39,116.0786 .62,35.76,31.83$, 31.33, 29.14, 22.73, 14.21. MS (MALDI-TOF): $m / z 1101.5\left(\mathrm{MH}^{+}\right)$. Anal. calc. for $\mathrm{C}_{72} \mathrm{H}_{76} \mathrm{O}_{2} \mathrm{~S}_{4}: \mathrm{C}$, 78.50; H, 6.95. Found: C, 78.58; H, 6.88.

NO4-CHO. This compound was prepared following the same procedure as for NO-CHO. The product was afforded as a yellow solid (220 mg, 95\%). ${ }^{1} \mathrm{H}$ NMR (400 MHz, $\left.\mathrm{CD}_{2} \mathrm{Cl}_{2}\right): \delta 9.81(\mathrm{~s}, 2 \mathrm{H})$, $7.98(\mathrm{~d}, J=8.8 \mathrm{~Hz}, 2 \mathrm{H}), 7.89(\mathrm{~s}, 2 \mathrm{H}), 7.98(\mathrm{~d}, J=8.8 \mathrm{~Hz}, 2 \mathrm{H}), 7.24(\mathrm{~d}, J=8.0 \mathrm{~Hz}, 8 \mathrm{H}), 7.12(\mathrm{~d}, J=$ $8.0 \mathrm{~Hz}, 8 \mathrm{H}), 2.57(\mathrm{t}, J=8.0 \mathrm{~Hz}, 8 \mathrm{H}), 1.56(\mathrm{~m}, 8 \mathrm{H}), 1.25(\mathrm{~m}, 24 \mathrm{H}), 0.85(\mathrm{~m}, 12 \mathrm{H}) .{ }^{13} \mathrm{C}$ NMR $(75$ $\left.\mathrm{MHz}, \mathrm{CDCl}_{3}\right): \delta 182.87,147.74,146.07,144.77,143.63,141.43,138.97,136.83,128.85,128.27$, $128.06,126.35,121.26,116.84,115.76,86.53,35.60,31.66,31.11,29.02,22.57,14.09 . \mathrm{MS}$ (MALDI-TOF): $m / z$ 1156.5 $\left(\mathrm{M}^{+}\right)$. Anal. calc. for $\mathrm{C}_{74} \mathrm{H}_{76} \mathrm{O}_{4} \mathrm{~S}_{4}: \mathrm{C}, 76.78 ; \mathrm{H}, 6.62$. Found: $\mathrm{C}, 76.67 ; \mathrm{H}$, 6.74 .

NOIC4. This compound was prepared following the same procedure as for NOIC. The product was afforded as a purple solid (256 mg, 81\%). ${ }^{1} \mathrm{H}$ NMR (300 MHz, $\left.\mathrm{CD}_{2} \mathrm{Cl}_{2}\right): \delta 8.72(\mathrm{~s}, 2 \mathrm{H}), 8.48(\mathrm{~m}$, 2H), $8.17(\mathrm{~s}, 2 \mathrm{H}), 8.02(\mathrm{~d}, J=9.0 \mathrm{~Hz}, 2 \mathrm{H}), 7.61(\mathrm{~m}, 2 \mathrm{H}), 7.49(\mathrm{~d}, J=9.0 \mathrm{~Hz}, 2 \mathrm{H}), 7.29(\mathrm{~d}, J=7.8$ 
$\mathrm{Hz}, 8 \mathrm{H}), 7.16(\mathrm{~d}, J=7.8 \mathrm{~Hz}, 8 \mathrm{H}), 2.58(\mathrm{t}, J=7.8 \mathrm{~Hz}, 8 \mathrm{H}), 1.55(\mathrm{~m}, 8 \mathrm{H}), 1.24(\mathrm{~m}, 24 \mathrm{H}), 0.80(\mathrm{~m}$, 12H). ${ }^{13} \mathrm{C} \mathrm{NMR}\left(75 \mathrm{MHz}, \mathrm{CDCl}_{3}\right): \delta 185.26,158.45,156.37,156.19,153.84,152.88,152.69,148.80$, $145.01,144.08,139.84,138.89,138.77,138.30,136.66,136.21,134.53,129.65,128.58,128.28$, $126.82,122.11,121.69,117.49,116.366,115.15,114.87,114.35,114.20,112.80,112.55,86.86$, 69.97, 53.58, 35.82, 31.88, 31.42, 29.18, 22.72, 14.20. MS (MALDI-TOF): $m / z$ 1581.1 $\left(\mathrm{M}^{+}\right)$. Anal. calc. for $\mathrm{C}_{98} \mathrm{H}_{80} \mathrm{~F}_{4} \mathrm{~N}_{4} \mathrm{O}_{4} \mathrm{~S}_{4}: \mathrm{C}, 74.41 ; \mathrm{H}, 5.10 ; \mathrm{N}, 3.54$. Found: C, 74.52; H, 5.05; N, 3.48.

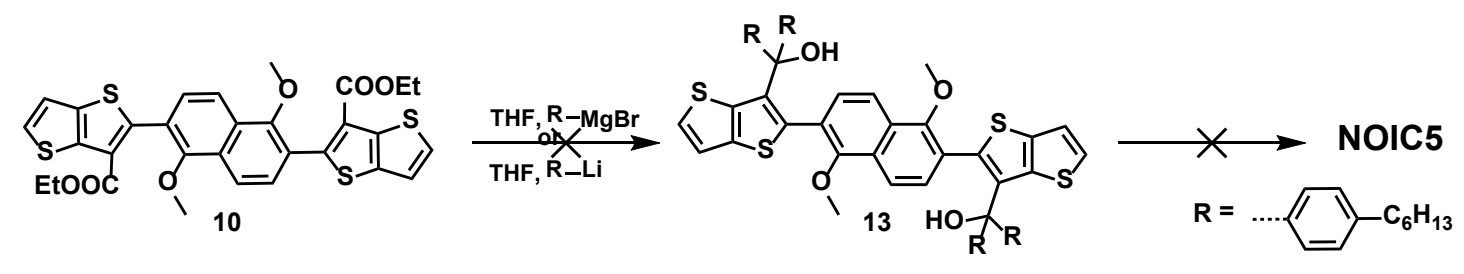

Scheme S7. Synthetic route for NOIC5

Compound 13. This compound was attempt to be obtained through regular nucleophilic addition reactions, using Grignard reagent or (4-hexylphenyl)lithium. However, no target product was obtained after reaction. Therefore, NOIC5 was not obtained finally.

\section{General characterization}

The ${ }^{1} \mathrm{H}$ and ${ }^{13} \mathrm{C}$ NMR spectra were obtained using a Bruker AVANCE $300 \mathrm{MHz}$ and $400 \mathrm{MHz}$ spectrometer. Mass spectra were recorded using AB Sciex 5800 MALDI-TOF/TOF Mass Spectrometer. Elemental analysis was performed using a Flash EA1112 analyzer. Thermogravimetric analysis measurements were performed using a thermogravimetric analyzer (Q600 TGA-DSC-DTA) under flowing nitrogen gas at a heating rate of $10{ }^{\circ} \mathrm{C} \mathrm{min}^{-1}$. UV-vis absorption spectra (solution in $\mathrm{CHCl}_{3}$; thin film on quartz substrate) were measured using a $\mathrm{J} \Lambda \mathrm{SCO} \mathrm{V}-570$ spectrophotometer. The electrochemical properties of the NOIC series were investigated in deoxygenated anhydrous acetonitrile under nitrogen at a scan rate of $100 \mathrm{mV} \mathrm{s}^{-1}$ using $0.1 \mathrm{M}$ tetrakis( $n$-butyl)ammonium hexafluorophosphate as supporting electrolyte using a computer-controlled CHI660C 
electrochemical workstation, a glassy carbon working electrode, a platinum-wire auxiliary electrode, and an $\mathrm{Ag} / \mathrm{AgCl}$ reference electrode. NOIC series films were drop-cast onto the working electrode from a $10 \mathrm{mg} \mathrm{mL}^{-1} \mathrm{CHCl}_{3}$ solution. A ferrocene/ferrocenium $\left(\mathrm{FeCp}_{2}{ }^{0 /+}\right)$ redox couple was used as internal standard and was assigned an absolute energy of $-4.8 \mathrm{eV}$ vs vacuum. The $\mathrm{FeCp}{ }_{2}{ }^{0 /+}$ redox couple was found at $0.431 \mathrm{~V}$ relative to the $\mathrm{Ag} / \mathrm{AgCl}$ electrode.

\section{Single crystal X-ray diffraction}

Single crystals of NOIC series compounds were grown by liquid transfer method. Ethanol was selected as poor solvent to diffuse slowly into closely saturated $\mathrm{CHCl}_{3}$ solution in one week. Single crystals were selected and quickly transferred into nitrogen flow at $150 \mathrm{~K}$. The diffraction signals were collected on Rigaku XtaLAB P2000 FR-X diffractometer using $\mathrm{CuK} \alpha$ radiation $(\lambda=1.54184$ $\AA$ ). Structure was solved by intrinsic phasing method $\left(\mathrm{SHELXT}^{\mathrm{S} 3}\right)$ and refined by least squares method $\left(\mathrm{SHELXL}^{\mathrm{S} 4}\right)$ integrated in Olex2. ${ }^{\mathrm{S} 5}$ Some electron density peaks were regarded as disordered composition like solvent due to the dynamical alkyl chains, and masked in the final refinement. We believed atomic positions in the conjugated backbone were acceptable for the structural analysis. Alykl chains were omitted when calculating packing index and void percentage using PLATONS. ${ }^{\text {S6 }}$

\section{Morphological characterization}

The surface morphology of the blend films was observed using an atomic force microscope (Dimension Icon, Bruker) in the standard mode. GIWAXS characterization of active layer was performed at beamline 7.3.3, Advanced Light Source (ALS), Lawrence Berkeley National Lab (LBNL). X-ray energy was $10 \mathrm{keV}$ and operated in top off mode. The scattering intensity was recorded on a 2D image plate (Pilatus $2 \mathrm{M}$ ) with a pixel size of $0.172 \mathrm{~mm}$ by $0.172 \mathrm{~mm}$. The samples were $\sim 10 \mathrm{~mm}$ long in the direction of the beam path, and the detector was located at a distance of 
$270 \mathrm{~mm}$ from the sample center (distance calibrated by AgB reference). The incidence angle was chosen to be $0.16^{\circ}$ (above critical angle) for GIWAXS measurement. ${ }^{\mathrm{S} 7-\mathrm{S} 9}$ OSC samples were prepared on PEDOT:PSS covered Si wafers in a similar manner to the OSC devices. The coherence length $\left(L_{\mathrm{c}}\right)$ of all the compounds were calculated from 1D profiles by Scherrer analysis ${ }^{\mathrm{S} 10}: L_{\mathrm{c}}=$ $2 \pi K / \Delta \mathrm{q}$, where $\Delta \mathrm{q}$ is the full-width at half-maximum of the peak and $K$ is a shape factor $(0.9$ was used here). R-SoXS was performed at beamline 11.0.1.2, ALS, LBNL. OSC samples for R-SoXS measurements were prepared on a PEDOT:PSS modified Si substrate under the same conditions as used for OSC device fabrication, and then transferred by floating in water to a $1.5 \mathrm{~mm} \times 1.5 \mathrm{~mm}, 100$ $\mathrm{nm}$ thick $\mathrm{Si}_{3} \mathrm{~N}_{4}$ membrane supported by a $5 \mathrm{~mm} \times 5 \mathrm{~mm}, 200 \mathrm{~mm}$ thick Si frame (Norcada Inc.).

\section{PL measurements}

PL spectra were measured using an Andor spectral graph (Kymera 328i) and a Newton CCD camera (DU970P-BVF). The lamp intensity was calibrated using a reference Si diode, and the current signal was amplified with a Stanford SR570 pre-amplifier.

\section{EL measurements}

EL measurements were conducted by direct-current meter (PWS2326, Tectronix) to provide bias voltage for the test device, and the EL spectra were recorded by the fluorescence spectrometer (KYMERA-328I-B2, Andor technology LTD) with cooled silicon array and indium gallium arsenic detectors, which was calibrated by standard light source (Ocean Optics).

\section{$\mathrm{EQE}_{\mathrm{EL}}$ measurements}

The $\mathrm{EQE}_{\mathrm{EL}}$ was recorded with an in-house-built system comprising a standard silicon photodiode (S1337-1010BR, Hamamatsu Electronics), Keithley 2400 source meter (for supplying voltages and recording injected current), and Keithley 6482 picoammeter (for measuring the emitting light 
intensity).

\section{SEQE measurements}

A $150 \mathrm{~W}$ quartz halogen lamp (LSH-75, Newport) acted as a light source, passing through the monochromator (CS260-RG-3-MC-A, Newport) to provide an adjustable monochromatic light source for testing, and then emitted an optical signal at a $173 \mathrm{~Hz}$ frequency through the chopper (3502 Optical Chopper, Newport) and focused on the OSC devices. The current generated by the device was amplified by the front-end current amplifier (SR570, Stanford) to reduce the impact of the noise signal. The final signal was collected and analyzed by a Phase-locked Amplifier (SR830 DSP Lock-In Amplifier, Stanford).

\section{Device fabrication and characterization}

The inverted structure of OSCs was ITO/ZnO/active layer/ $\mathrm{MoO}_{3} / \mathrm{Ag}$. Patterned ITO glass (sheet resistance $=15 \Omega$ ) was precleaned in an ultrasonic bath with ultra-pure water, acetone and isopropanol, and treated in an ultraviolet-ozone chamber (Jelight Company, USA) for 3 min. ZnO layer (ca. $30 \mathrm{~nm}$ ) was spin-coated at $4000 \mathrm{rpm}$ onto the ITO glass from $\mathrm{ZnO}$ precursor solution (100 mg $\mathrm{Zn}\left(\mathrm{CH}_{3} \mathrm{COO}\right)_{2} \cdot 2 \mathrm{H}_{2} \mathrm{O}$ and $28.29 \mu \mathrm{L}$ ethanolamine dissolved in $973 \mu \mathrm{L}$ 2-methoxyethanol), and then baked at $200{ }^{\circ} \mathrm{C}$ for 30 min. PM6 and NOIC series (1:1, weight ratio) were dissolved in $\mathrm{CHCl}_{3}$ (12.5 $\mathrm{mg} \mathrm{mL}^{-1}$ in total) with $0.2 \mathrm{vol} \%$ of DIO as an additive and the spin-coated films (2000 rpm) were annealed at $110{ }^{\circ} \mathrm{C}$ for $10 \mathrm{~min}$ to form the photoactive layers. The $\mathrm{MoO}_{3}$ layer $(\mathrm{ca} .5 \mathrm{~nm})$ and Ag electrode $(c a .80 \mathrm{~nm})$ were slowly evaporated onto the surface of the photoactive layer under vacuum $\left(c a .10^{-5} \mathrm{~Pa}\right)$. The active area of the device was $c a .4 \mathrm{~mm}^{2}$. The devices were not masked and the active area of devices was measured by optical microscopy. The $J-V$ curve was measured using a computer-controlled B2912A Precision Source/Measure Unit (Agilent Technologies). An XES-70S1 
(SAN-EI Electric Co., Ltd.) solar simulator (AAA grade, $70 \times 70 \mathrm{~mm}^{2}$ photobeam size) coupled with AM 1.5 G solar spectrum filters was used as the light source, and the optical power at the sample was $100 \mathrm{~mW} \mathrm{~cm}^{-2}$. A $2 \mathrm{~cm} \times 2 \mathrm{~cm}$ monocrystalline silicon reference cell (SRC-1000-TC-QZ) was purchased from VLSI Standards Inc. The EQE spectrum was measured using Solar Cell Spectral Response Measurement System QE-R3011 (Enlitech Co., Ltd.). The light intensity at each wavelength was calibrated using a standard single crystal Si photovoltaic cell. For light stability test, we measured the photovoltaic performance of the unencapsulated devices under continuous AM 1.5G illumination at $100 \mathrm{~mW} \mathrm{~cm} \mathrm{~cm}^{-2}$ in glove box for a certain time. For thermal stability test, we heated the unencapsulated devices at $100{ }^{\circ} \mathrm{C}$ in glove box for a certain time and measured the photovoltaic performance of the devices under AM $1.5 \mathrm{G}$ illumination at $100 \mathrm{~mW} \mathrm{~cm}$.

\section{Mobility measurements}

Electron-only devices were fabricated using the architectures of ITO/ZnO/acceptor or PM6:acceptor/Ca/Al. $15 \mathrm{mg} \mathrm{mL}^{-1}$ acceptor in $\mathrm{CHCl}_{3}$ or $12.5 \mathrm{mg} \mathrm{mL}^{-1} \mathrm{PM6}$ :acceptor mixture in $\mathrm{CHCl}_{3}$ treated with optimal device condition was spin-coated as active layer $(c a .100 \mathrm{~nm})$, and then $\mathrm{Ca}(c a .10 \mathrm{~nm})$ and $\mathrm{Al}(c a .60 \mathrm{~nm})$ were evaporated under vacuum $\left(c a .10^{-5} \mathrm{~Pa}\right)$. Hole-only devices were fabricated using the architecture of ITO/PEDOT:PSS/PM6:acceptor/Au. The pre-cleaned ITO glass was spin-coated with PEDOT:PSS (ca. $30 \mathrm{~nm}$ ), then $12.5 \mathrm{mg} \mathrm{mL}^{-1}$ PM6:acceptor mixture in $\mathrm{CHCl}_{3}$ treated with optimal device condition was spin-coated as active layer $(c a .100 \mathrm{~nm})$, and then $\mathrm{Au}(c a .30 \mathrm{~nm})$ was evaporated under vacuum $\left(c a \cdot 10^{-5} \mathrm{~Pa}\right)$ at a low speed $(1 \AA / 5 \mathrm{~s})$ to avoid the penetration of $\mathrm{Au}$ atoms into the active layer. The mobility was extracted by fitting the current density-voltage curves using SCLC. ${ }^{\mathrm{S} 11}$ The formula is as follows.

$$
J=\frac{9}{8} \mu \varepsilon_{0} \varepsilon_{r} \frac{V^{2}}{d^{3}} e^{0.89 \sqrt{\frac{V}{E_{0} d}}}
$$


where $J$ is current density, $\mu$ is hole or electron mobility, $\varepsilon_{\mathrm{r}}$ is relative dielectric constant, $\varepsilon_{0}$ is permittivity of free space, $V=V_{\mathrm{appl}}-V_{\mathrm{bi}}$, where $V_{\mathrm{appl}}$ is the applied voltage to the device, and $V_{\mathrm{bi}}$ is the built-in voltage due to the difference in work function of the two electrodes (for hole-only diodes, $V_{\mathrm{bi}}$ is $0.2 \mathrm{~V}$; for electron-only diodes, $V_{\mathrm{bi}}$ is $\left.0 \mathrm{~V}\right) . E_{0}$ is characteristic field, $d$ is the thickness of organic layer. The thickness of organic layer was measured on DektakXT (Bruker). The $J-V$ curves of the devices are plotted as $\ln \left[J d^{3} /\left(V_{\mathrm{appl}}-V_{\mathrm{bi}}\right)^{2}\right]$ versus $\left[\left(V_{\mathrm{appl}}-V_{\mathrm{bi}}\right) / d\right]^{0.5}$.

\section{Voltage loss calculation}

According to detailed balance theory, $V_{\mathrm{OC}}$ of a $\mathrm{BHJ}$ solar cell is determined by $E_{\mathrm{CT}}$, radiative, and non-radiative recombination losses of charge carriers: ${ }^{\mathrm{S} 12}$

$$
V_{\text {loss }}=\frac{E_{\mathrm{g}}^{\mathrm{PV}}}{q}-V_{\mathrm{OC}}=\frac{E_{\mathrm{g}}^{\mathrm{PV}}-E_{\mathrm{CT}}}{q}+\frac{k_{\mathrm{B}} T}{q} \ln \left(\frac{1}{\mathrm{EQE}_{\mathrm{EL}}}\right)+\Delta V_{\mathrm{rad}}=\frac{\Delta E_{\mathrm{CT}}}{q}+\Delta V_{\mathrm{nonrad}}+\Delta V_{\mathrm{rad}}
$$

$q$ is the elementary charge, $E_{\mathrm{g}}{ }^{\mathrm{PV}}$ is the bandgap of the blend film, $k_{\mathrm{B}}$ is the Boltzmann constant, $T$ is the absolute temperature in Kelvin, $\mathrm{EQE}_{\mathrm{EL}}$ is the $\mathrm{EQE}$ of $\mathrm{EL}, \Delta V_{\mathrm{rad}}$ is the radiative recombination voltage loss, $\Delta E_{\mathrm{CT}}$ is defined as the offset between $E_{\mathrm{g}}{ }^{\mathrm{PV}}$ and $E_{\mathrm{CT}}, \Delta V_{\text {nonrad }}$ represents the non-radiative recombination voltage loss. 

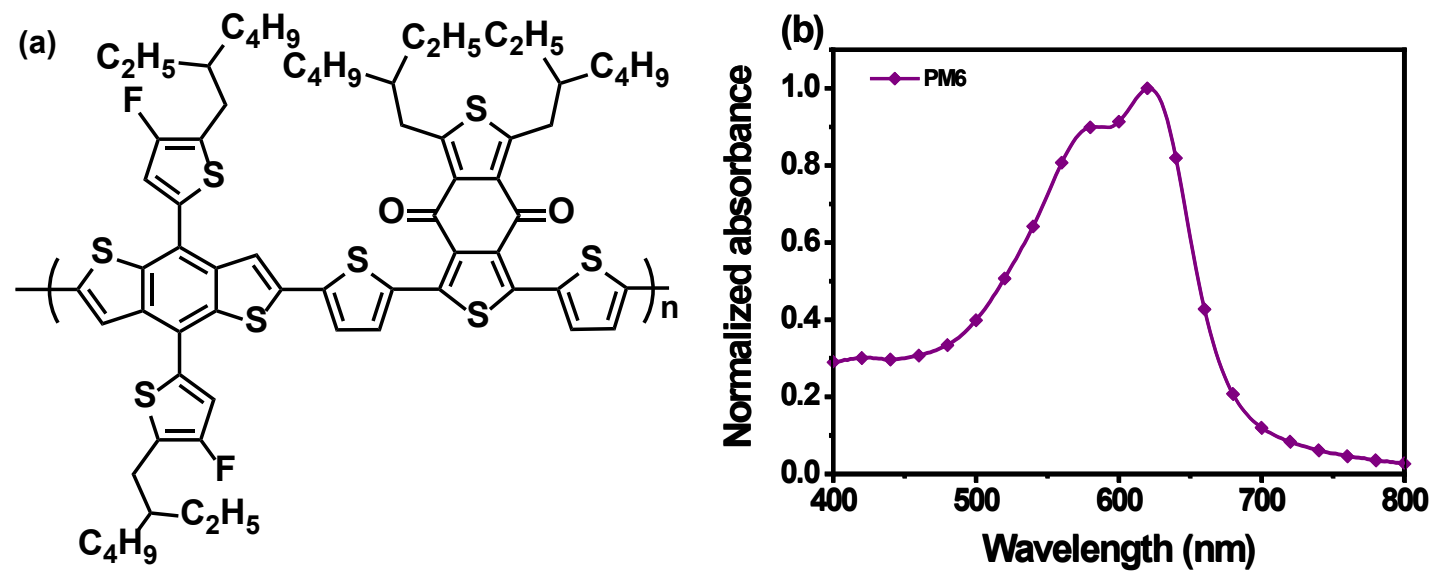

Figure S1. (a) The chemical structure of PM6. (b) The absorption spectrum of PM6 as a thin film.

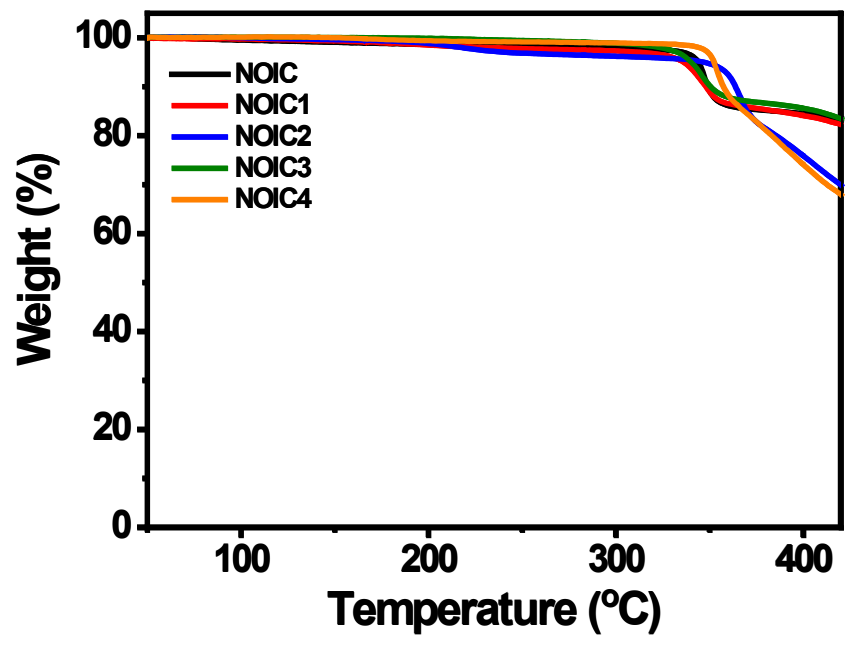

Figure S2. TGA curves of NOIC series.

(a)

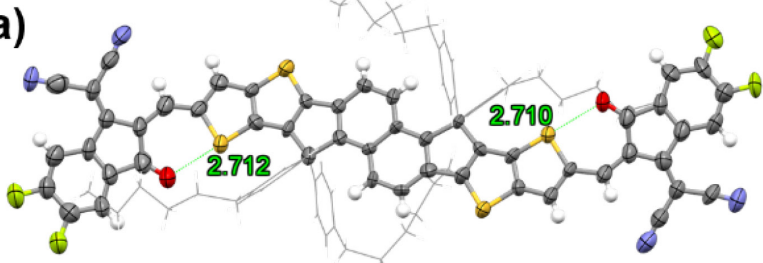

(c)

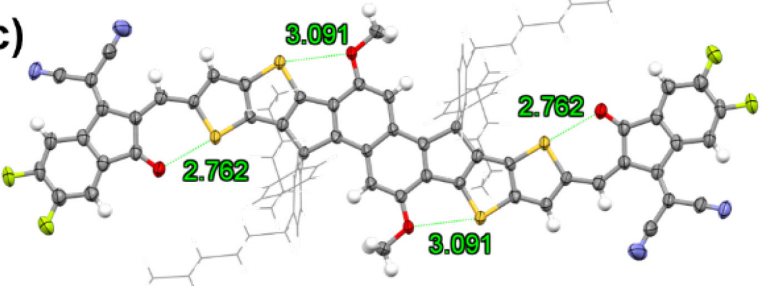

(b)

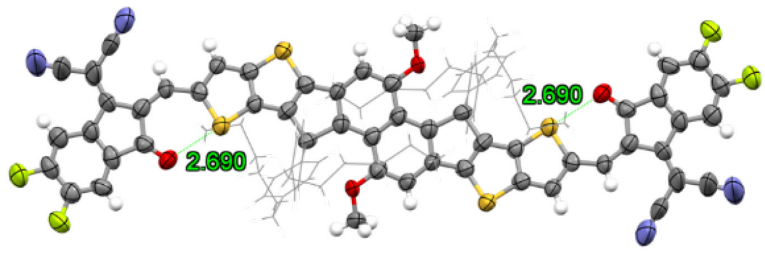

(d)

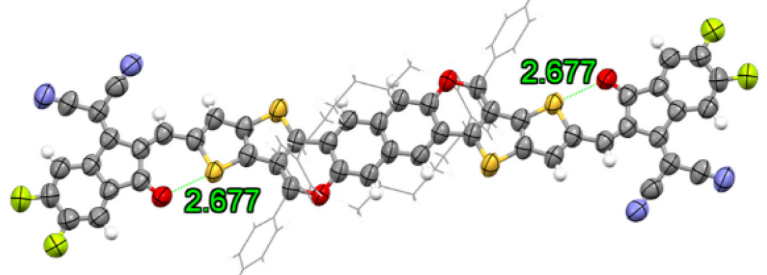

Figure S3. Molecular conformations of (a) NOIC, (b) NOIC1, (c) NOIC2 and (d) NOIC3 in the crystal structures. 

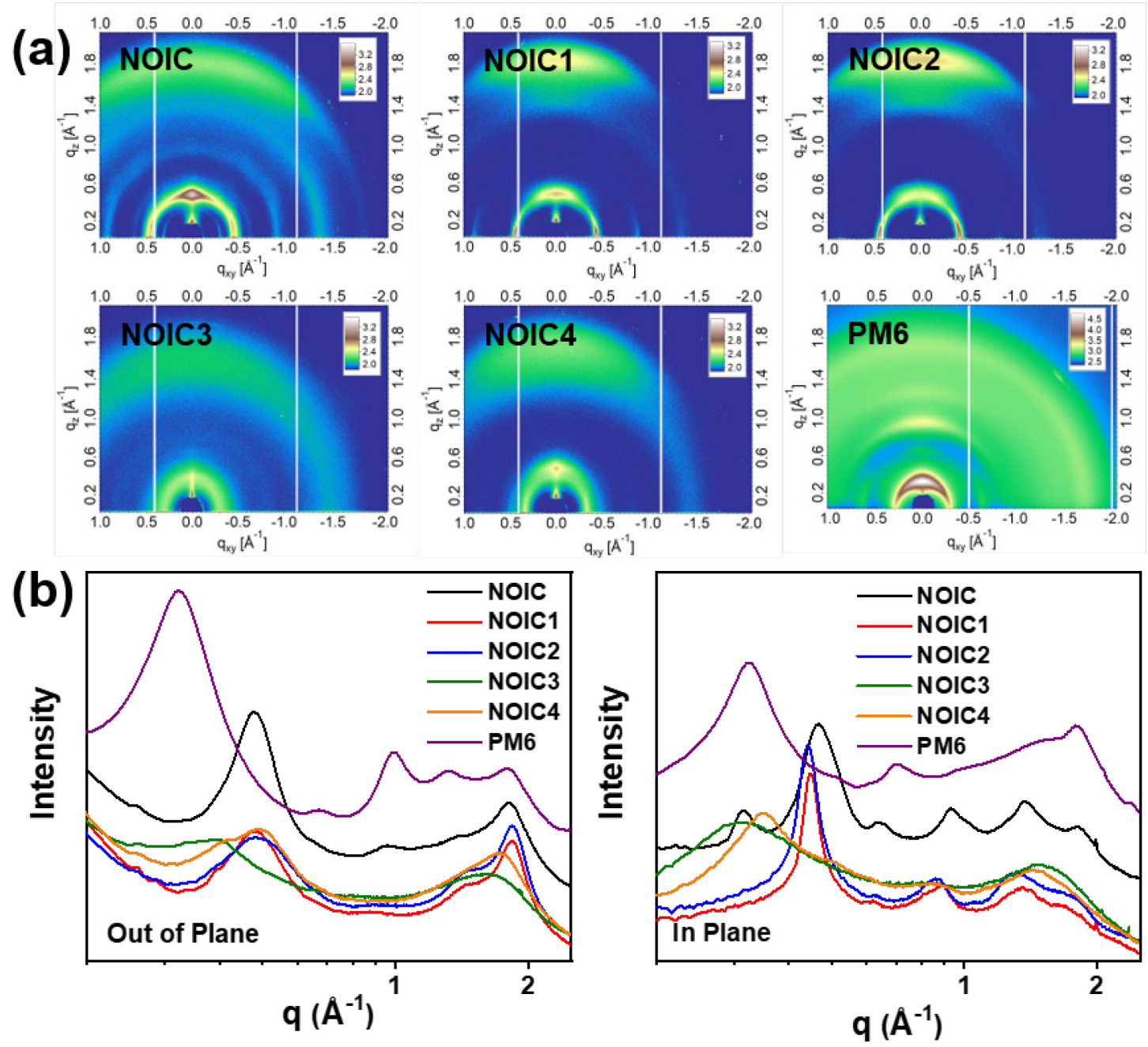

Figure S4. (a) 2D GIWAXS patterns, and (b) scattering profiles of out-of-plane and in-plane for PM6 and NOIC series neat films.
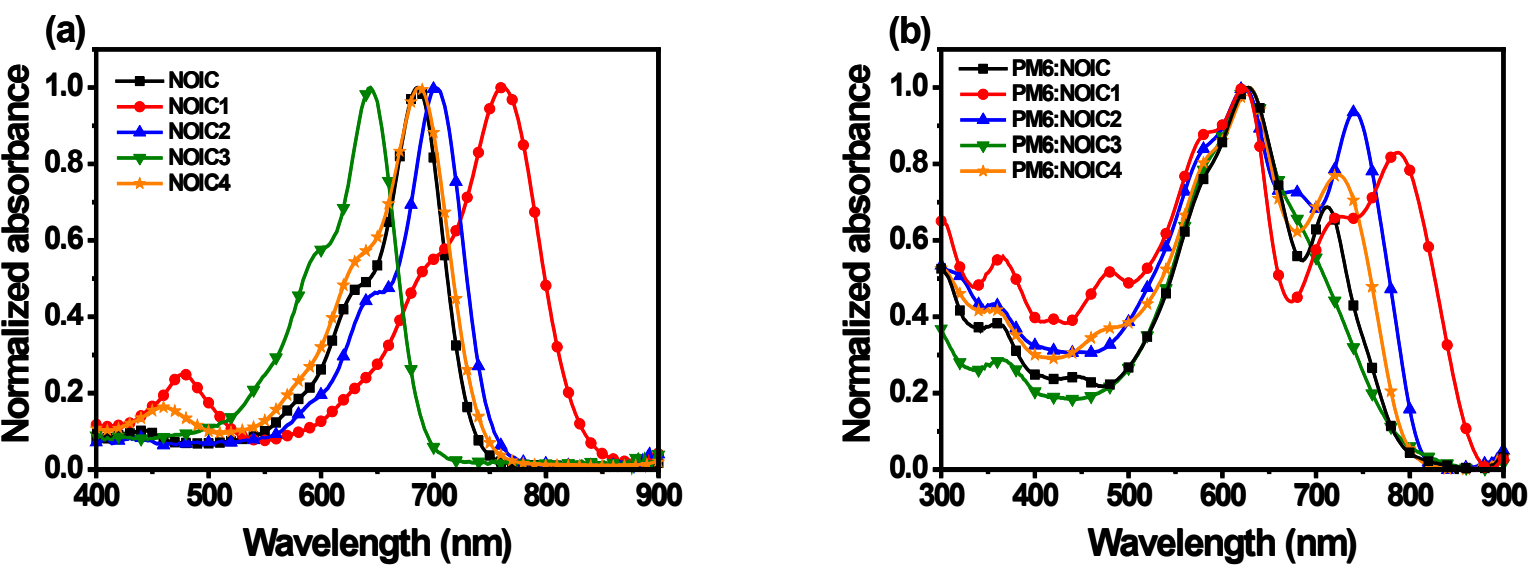

Figure S5. Absorption spectra of (a) NOIC series in $\mathrm{CHCl}_{3}$ solution and (b) PM6:NOIC series as thin films. 


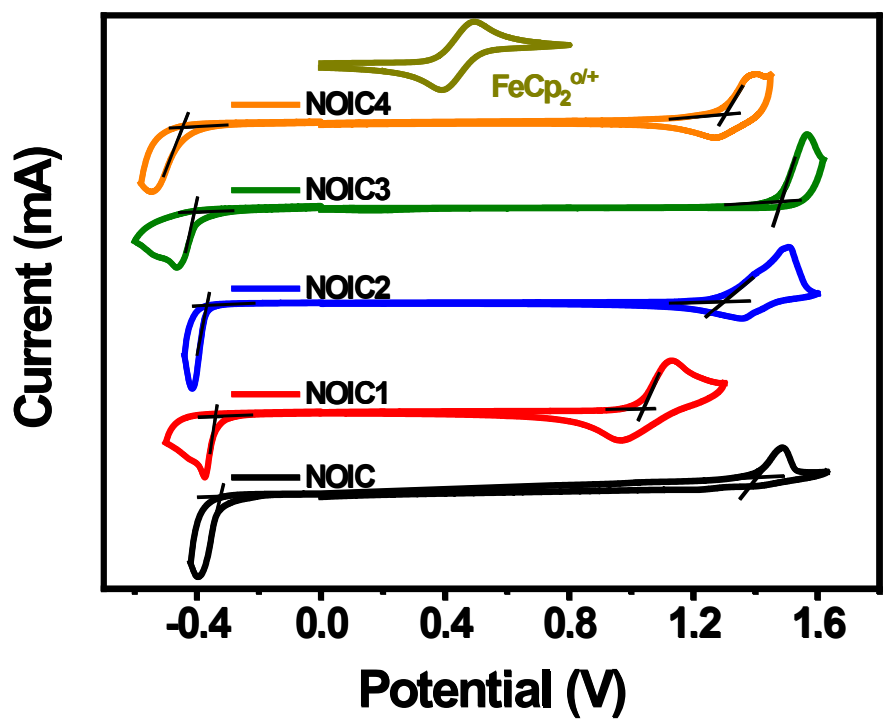

Figure S6. Cyclic voltammograms of NOIC series.

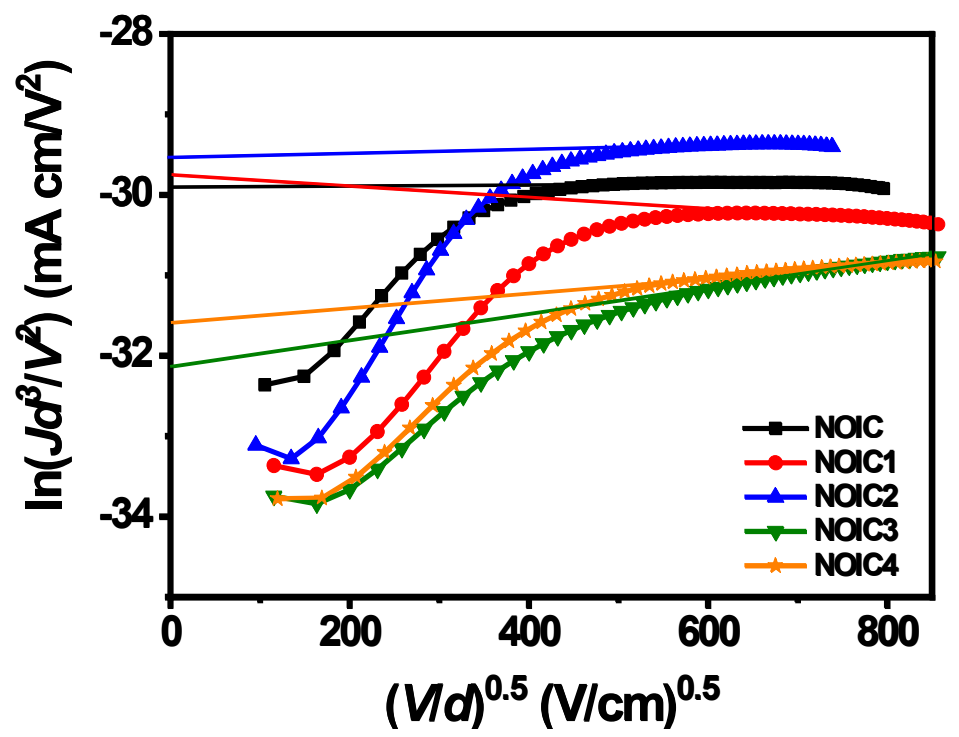

Figure S7. $J-V$ characteristics in the dark for electron-only devices based on NOIC series. 


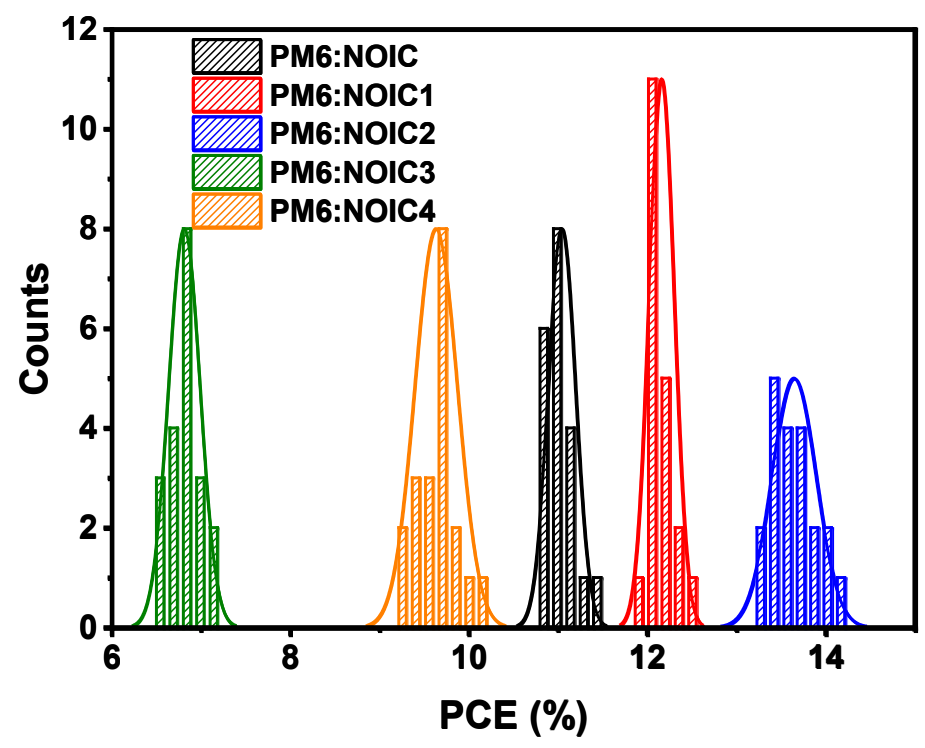

Figure S8. Statistical histogram of PCEs of the optimized devices based on PM6:NOIC series.

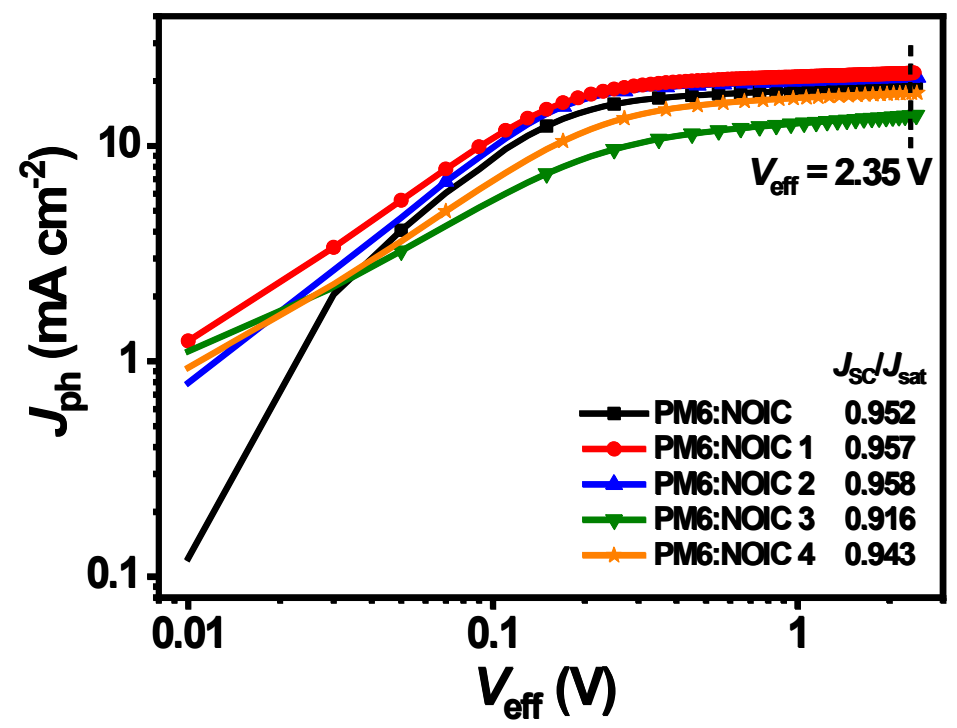

Figure S9. $J_{\text {ph }}$ versus $V_{\text {eff }}$ characteristics of the devices based on PM6:NOIC series. 

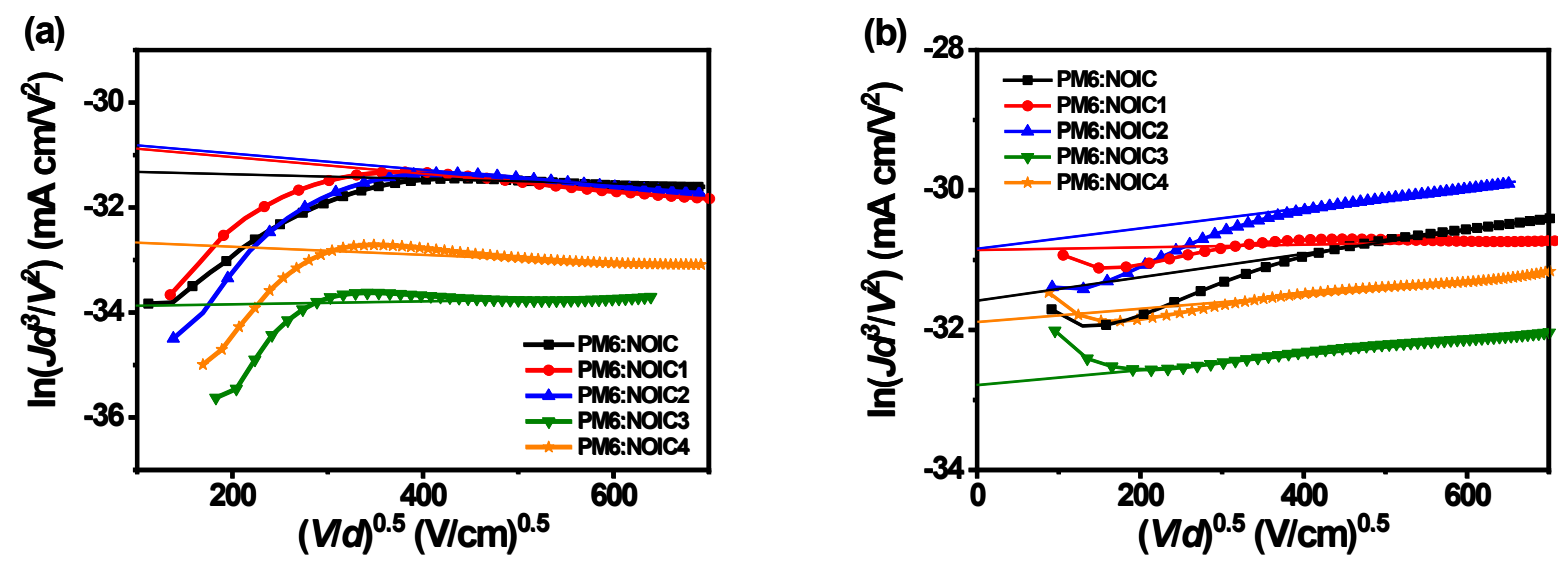

Figure S10. $J-V$ characteristics in the dark for (a) hole-only and (b) electron-only devices based on PM6:NOIC series.
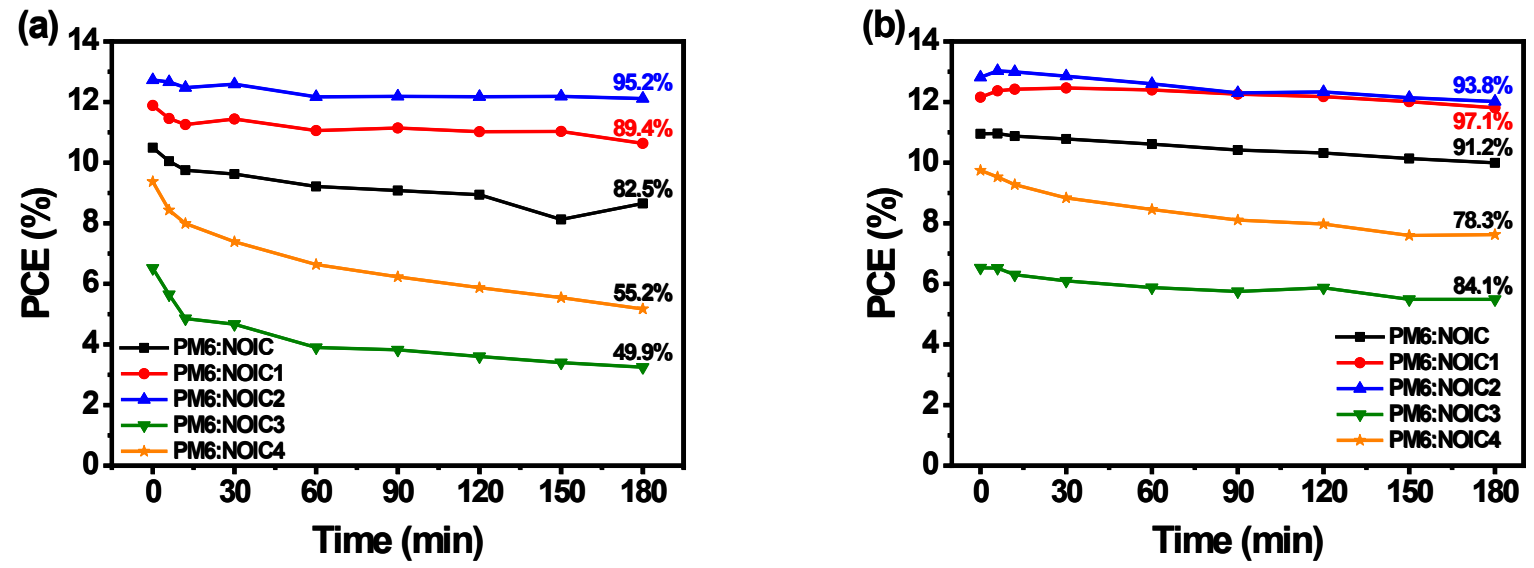

Figure S11. Stability of the devices based on PM6:NOIC series under (a) AM 1.5G illumination at $100 \mathrm{~mW} \mathrm{~cm}{ }^{-2}$ and (b) heating at $100{ }^{\circ} \mathrm{C}$.
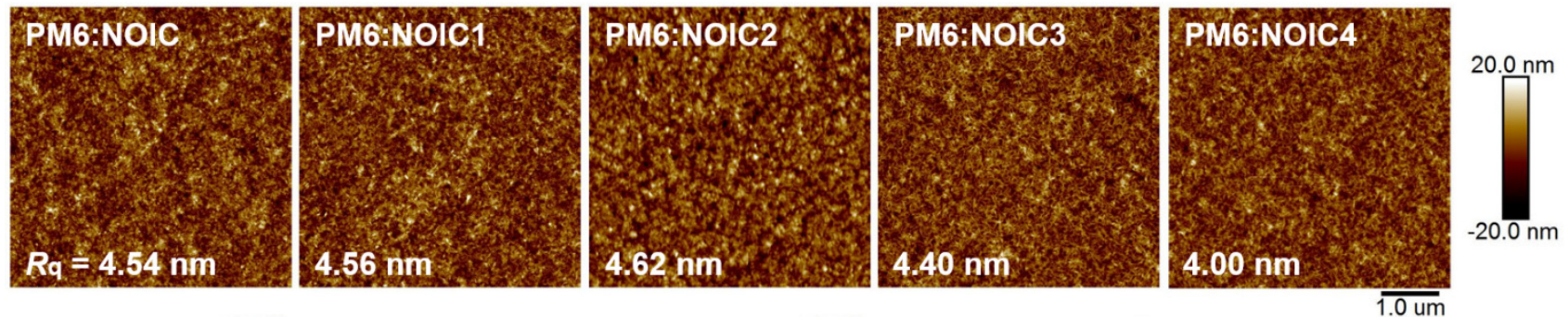

Figure S12. AFM height images of PM6:NOIC series blend films. 


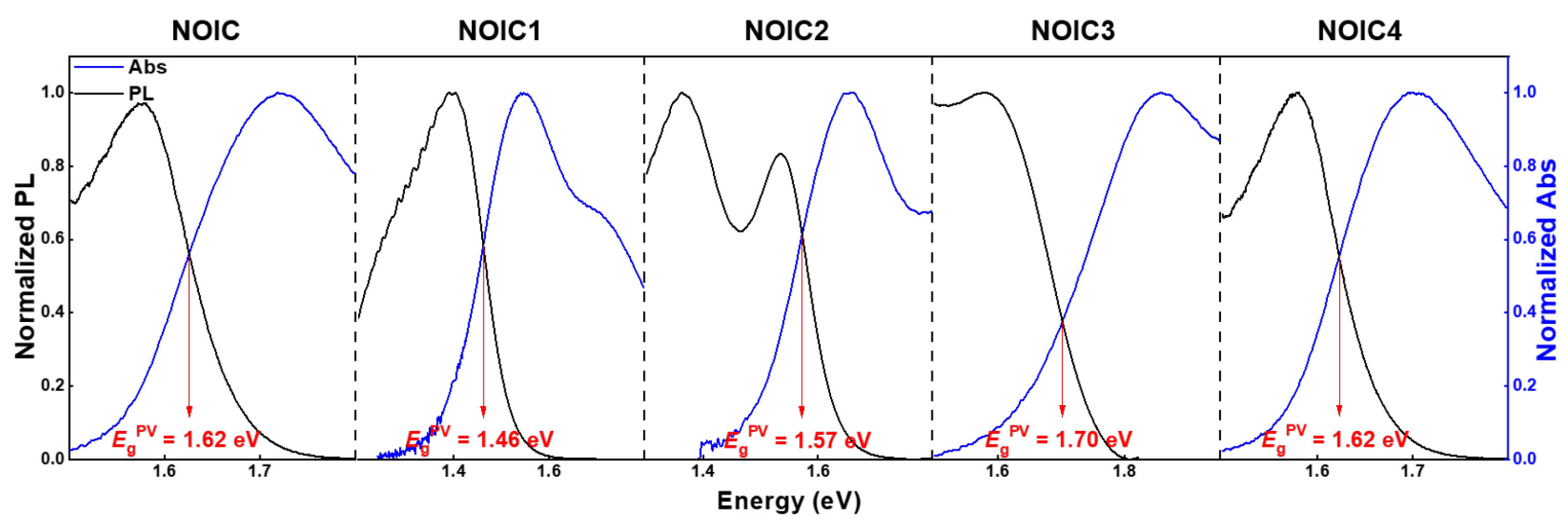

Figure S13. Normalized PL and absorption (abs) spectra of the pure NOIC series films.

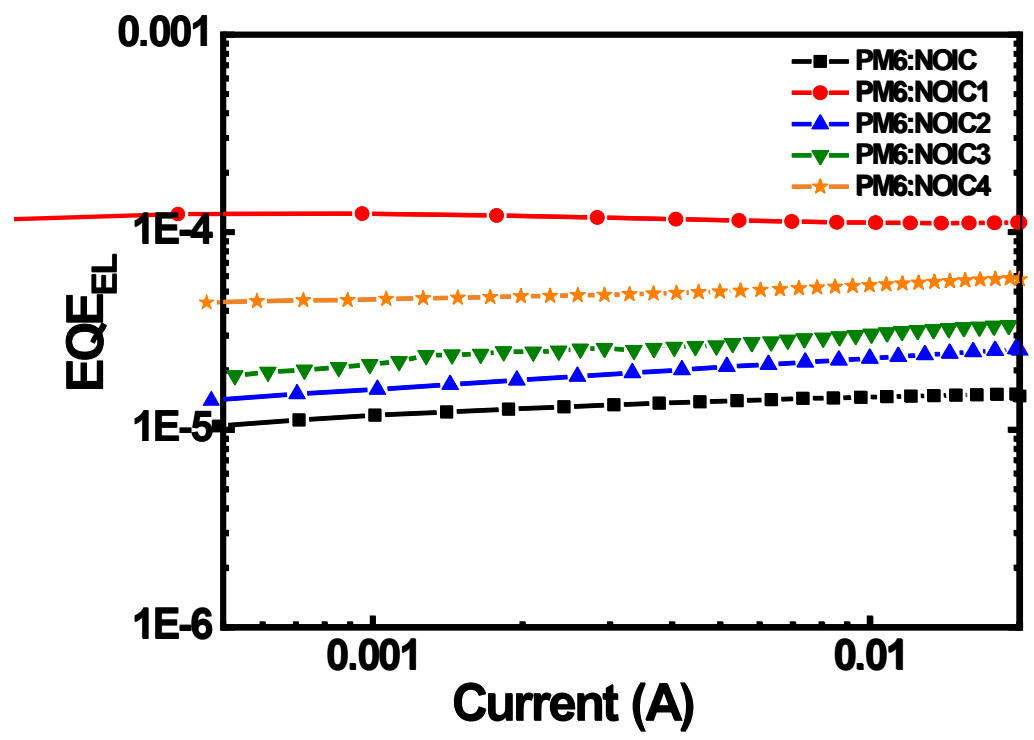

Figure S14. $\mathrm{EQE}_{\mathrm{EL}}$ of optimal OSCs based on PM6:NOIC series. 
Table S1. Crystal data and structure refinement for NOIC series compounds.

\begin{tabular}{|c|c|c|c|c|}
\hline Identification code & NOIC & NOIC1 & NOIC2 & NOIC3 \\
\hline CCDC number & 2031646 & 2031647 & 2031648 & 2031649 \\
\hline Temperature/K & $150.00(10)$ & 149.99(10) & $149.99(10)$ & $149.99(10)$ \\
\hline Crystal system & triclinic & triclinic & triclinic & triclinic \\
\hline Space group & $\mathrm{P}-1$ & $\mathrm{P}-1$ & P-1 & $\mathrm{P}-1$ \\
\hline $\mathrm{a} / \AA$ & $14.5156(8)$ & $17.0807(10)$ & $13.8062(9)$ & $18.5031(16)$ \\
\hline $\mathrm{b} / \AA \AA$ & $21.1136(10)$ & $18.5235(11)$ & $15.6264(10)$ & $19.1175(13)$ \\
\hline $\mathrm{c} / \AA$ & $27.8392(16)$ & $31.1202(19)$ & $21.4134(6)$ & $20.2903(16)$ \\
\hline$\alpha /^{\circ}$ & $109.648(5)$ & $83.407(5)$ & $103.743(4)$ & $70.746(7)$ \\
\hline$\beta /{ }^{\circ}$ & $91.109(5)$ & $87.705(5)$ & $94.765(4)$ & $73.068(7)$ \\
\hline$\gamma /{ }^{\circ}$ & $99.314(4)$ & $62.786(6)$ & $112.984(6)$ & $65.994(8)$ \\
\hline Volume $/ \AA^{3}$ & $7904.3(8)$ & $8697.6(10)$ & $4050.6(4)$ & $6086.3(10)$ \\
\hline Z & 4 & 4 & 2 & 3 \\
\hline$\rho_{\text {calc }} \mathrm{g} / \mathrm{cm}^{3}$ & 1.302 & 1.218 & 1.32 & 1.295 \\
\hline$\mu / \mathrm{mm}^{-1}$ & 1.621 & 1.505 & 1.621 & 1.609 \\
\hline $\mathrm{F}(000)$ & 3248 & 3338 & 1688 & 2484 \\
\hline Crystal size $/ \mathrm{mm}^{3}$ & $0.11 \times 0.08 \times 0.04$ & $0.1 \times 0.08 \times 0.04$ & $0.1 \times 0.08 \times 0.04$ & $0.08 \times 0.06 \times 0.04$ \\
\hline Radiation & \multicolumn{4}{|c|}{$\operatorname{CuK} \alpha(\lambda=1.54184)$} \\
\hline $2 \Theta$ range for data collection ${ }^{\circ}$ & 6.19 to 91.648 & 5.396 to 134.15 & 6.612 to 134.146 & 5.214 to 134.146 \\
\hline Index ranges & $\begin{array}{l}-13 \leq \mathrm{h} \leq 13 \\
-19 \leq \mathrm{k} \leq 19 \\
25 \leq 1 \leq 25\end{array}$ & $\begin{array}{l}-20 \leq \mathrm{h} \leq 20 \\
-21 \leq \mathrm{k} \leq 22 \\
-37 \leq 1 \leq 34\end{array}$ & $\begin{array}{l}-16 \leq \mathrm{h} \leq 16 \\
-17 \leq \mathrm{k} \leq 18 \\
-25 \leq 1 \leq 22\end{array}$ & $\begin{array}{l}-21 \leq \mathrm{h} \leq 22 \\
-12 \leq \mathrm{k} \leq 22 \\
-21 \leq 1 \leq 24\end{array}$ \\
\hline Reflections collected & 56659 & 74363 & 39396 & 60193 \\
\hline Independent reflections & $\begin{array}{l}13019 \\
{\left[\mathrm{R}_{\text {int }}=0.1107\right.} \\
\left.\mathrm{R}_{\text {sigma }}=0.1105\right]\end{array}$ & $\begin{array}{l}29410 \\
{\left[\mathrm{R}_{\text {int }}=0.1156,\right.} \\
\left.\mathrm{R}_{\text {sigma }}=0.1822\right]\end{array}$ & $\begin{array}{l}14138 \\
{\left[\mathrm{R}_{\text {int }}=0.1403,\right.} \\
\left.\mathrm{R}_{\text {sigma }}=0.1722\right]\end{array}$ & $\begin{array}{l}21238 \\
{\left[\mathrm{R}_{\text {int }}=0.1388,\right.} \\
\left.\mathrm{R}_{\text {sigma }}=0.1920\right]\end{array}$ \\
\hline Data/restraints/parameters & $13019 / 146 / 2025$ & $29410 / 229 / 2058$ & $14138 / 14 / 1145$ & $21238 / 513 / 1546$ \\
\hline Goodness-of-fit on $\mathrm{F}^{2}$ & 1.027 & 1.022 & 0.957 & 0.934 \\
\hline Final R indexes $[\mathrm{I}>=2 \sigma(\mathrm{I})]$ & $\begin{array}{l}\mathrm{R}_{1}=0.0951 \\
\mathrm{wR}_{2}=0.2472\end{array}$ & $\begin{array}{l}\mathrm{R}_{1}=0.1364 \\
\mathrm{wR}_{2}=0.3338\end{array}$ & $\begin{array}{l}\mathrm{R}_{1}=0.0821 \\
\mathrm{wR}_{2}=0.1751\end{array}$ & $\begin{array}{l}\mathrm{R}_{1}=0.1048 \\
\mathrm{wR}_{2}=0.2554\end{array}$ \\
\hline Final $\mathrm{R}$ indexes [all data] & $\begin{array}{l}\mathrm{R}_{1}=0.1811 \\
\mathrm{wR}_{2}=0.3091\end{array}$ & $\begin{array}{l}\mathrm{R}_{1}=0.2892 \\
\mathrm{wR}_{2}=0.4320\end{array}$ & $\begin{array}{l}\mathrm{R}_{1}=0.1940 \\
\mathrm{wR}_{2}=0.2300\end{array}$ & $\begin{array}{l}\mathrm{R}_{1}=0.2879 \\
\mathrm{wR}_{2}=0.3677\end{array}$ \\
\hline
\end{tabular}

Table S2. Packing index and void percentage for single crystals of NOIC series compounds.

\begin{tabular}{ccc}
\hline & Packing index $^{a}$ & Void percentage $^{a}$ \\
\hline NOIC & $48.10 \%$ & $38.90 \%$ \\
NOIC1 & $45.70 \%$ & $41.70 \%$ \\
NOIC2 & $49.20 \%$ & $36.90 \%$ \\
NOIC3 & $47.40 \%$ & $36.60 \%$ \\
${ }^{a}$ Alkyl chains & are regarded as void and omitted when \\
calculating the packing index and void percentage.
\end{tabular}


Table S3. The optimization of the OSC devices based on PM6:NOIC series (D/A = 1:1, w/w).

\begin{tabular}{|c|c|c|c|c|c|}
\hline acceptor & optimization & $V_{\mathrm{OC}}(\mathrm{V})$ & $J_{\mathrm{SC}}\left(\mathrm{mA} \mathrm{cm}^{-2}\right)$ & FF $(\%)$ & PCE $(\%)$ \\
\hline \multirow{3}{*}{ NOIC } & as cast & 0.905 & 14.5 & 68.5 & 8.98 \\
\hline & $0.2 \% \mathrm{DIO}$ & 0.895 & 17.4 & 69.3 & 10.8 \\
\hline & $0.2 \% \mathrm{DIO}, 110^{\circ} \mathrm{C} 10 \mathrm{~min}$ & 0.885 & 18.1 & 71.2 & 11.4 \\
\hline \multirow{3}{*}{ NOIC1 } & as cast & 0.885 & 17.2 & 63.8 & 9.71 \\
\hline & $0.2 \% \mathrm{DIO}$ & 0.878 & 19.9 & 64.7 & 11.3 \\
\hline & $0.2 \% \mathrm{DIO}, 110^{\circ} \mathrm{C} 10 \mathrm{~min}$ & 0.864 & 21.9 & 65.9 & 12.5 \\
\hline \multirow{3}{*}{ NOIC2 } & as cast & 0.935 & 18.1 & 67.5 & 11.4 \\
\hline & $0.2 \% \mathrm{DIO}$ & 0.929 & 19.5 & 71.5 & 13.0 \\
\hline & $0.2 \% \mathrm{DIO}, 110^{\circ} \mathrm{C} 10 \mathrm{~min}$ & 0.927 & 20.6 & 73.8 & 14.1 \\
\hline \multirow{3}{*}{ NOIC3 } & as cast & 0.972 & 10.4 & 50.0 & 5.05 \\
\hline & $0.2 \% \mathrm{DIO}$ & 0.954 & 11.2 & 52.5 & 5.61 \\
\hline & $0.2 \%$ DIO, $110^{\circ} \mathrm{C} 10 \mathrm{~min}$ & 0.930 & 12.9 & 59.8 & 7.15 \\
\hline \multirow{3}{*}{ NOIC4 } & as cast & 0.964 & 14.1 & 49.9 & 6.78 \\
\hline & $0.2 \% \mathrm{DIO}$ & 0.964 & 15.0 & 55.9 & 8.10 \\
\hline & $0.2 \% \mathrm{DIO}, 110^{\circ} \mathrm{C} 10 \mathrm{~min}$ & 0.944 & 16.8 & 63.7 & 10.1 \\
\hline
\end{tabular}




\section{References}

(1) Kim, Y.-A.; Kang, M.; Jeon, Y.-J.; Hwang, K.; Kim, Y.-J.; Jang, S.-Y.; Kim, I.-B.; Kwon, G.; Kim, D.-Y. Structure-property relationship of D-A type copolymers based on phenanthrene and naphthalene units for organic electronics. J. Mater. Chem. C 2017, 5 (39), 10332-10342.

(2) Zhang, M.; Guo, X.; Ma, W.; Ade, H.; Hou, J. A large-bandgap conjugated polymer for versatile photovoltaic applications with high performance. Adv. Mater. 2015, 27 (31), 4655-4660.

(3) Dolomanov, O. V.; Bourhis, L. J.; Gildea, R. J.; Howard, J. A. K.; Puschmann, H. Olex2: A complete structure solution, refinement and analysis program. J. Appl. Cryst. 2009, 42 (2), 339-341.

(4) Sheldrick, G. M. Shelxt: Integrating space group determination and structure solution. Acta Cryst. 2014, A70, C1437.

(5) Sheldrick, G. M. Crystal structure refinement with shelxl. Acta Cryst. 2015, C71, 3-8.

(6) Spek, A. L. Single-crystal structure validation with the program platon. J. Appl. Cryst. 2003, 36 (1), 7-13.

(7) Ilavsky, J.; Jemian, P. R. Irena: Tool suite for modeling and analysis of small-angle scattering. $J$. Appl. Crystallogr. 2009, 42 (2), 347-353.

(8) Nelson, A. Co-refinement of multiple-contrast neutron/x-ray reflectivity data using motofit. $J$. Appl. Crystallogr. 2006, 39 (2), 273-276.

(9) Beaucage, G. Approximations leading to a unified exponential/power-law approach to small-angle scattering. J. Appl. Crystallogr. 1995, 28 (6), 717-728.

(10)Rivnay, J.; Noriega, R.; Kline, R. J.; Salleo, A.; Toney, M. F. Quantitative analysis of lattice disorder and crystallite size in organic semiconductor thin films. Phys. Rev. B: Condens. Matter Mater. Phys. 2011, 84 (4), 045203. 
(11)Malliaras, G. G.; Salem, J. R.; Brock, P. J.; Scott, C. Electrical characteristics and efficiency of single-layer organic light-emitting diodes. Phys. Rev. B: Condens. Matter Mater. Phys. 1998, 58 (20), No. R13411.

(12) Vandewal, K.; Tvingstedt, K.; Gadisa, A.; Inganäs, O.; Manca, J. V. Relating the open-circuit voltage to interface molecular properties of donor:Acceptor bulk heterojunction solar cells. Phys. Rev. B: Condens. Matter Mater. Phys. 2010, 81 (12), 125204. 